\title{
Gene family structure, expression and functional analysis of HD-Zip III genes in angiosperm and gymnosperm forest trees
}

\author{
Caroline L Côté ${ }^{1}$, Francis Boileau', Vicky Roy ${ }^{1}$, Mario Ouellet ${ }^{3}$, Caroline Levasseur ${ }^{2}$, Marie-Josée Morency², \\ Janice EK Cooke", Armand Séguin², John J MacKay ${ }^{1 *}$
}

\begin{abstract}
Background: Class III Homeodomain Leucine Zipper (HD-Zip III) proteins have been implicated in the regulation of cambium identity, as well as primary and secondary vascular differentiation and patterning in herbaceous plants. They have been proposed to regulate wood formation but relatively little evidence is available to validate such a role. We characterised and compared HD-Zip III gene family in an angiosperm tree, Populus spp. (poplar), and the gymnosperm Picea glauca (white spruce), representing two highly evolutionarily divergent groups.

Results: Full-length CDNA sequences were isolated from poplar and white spruce. Phylogenetic reconstruction indicated that some of the gymnosperm sequences were derived from lineages that diverged earlier than angiosperm sequences, and seem to have been lost in angiosperm lineages. Transcript accumulation profiles were assessed by RT-qPCR on tissue panels from both species and in poplar trees in response to an inhibitor of polar auxin transport. The overall transcript profiles HD-Zip III complexes in white spruce and poplar exhibited substantial differences, reflecting their evolutionary history. Furthermore, two poplar sequences homologous to HD-Zip III genes involved in xylem development in Arabidopsis and Zinnia were over-expressed in poplar plants. PtaHB1 overexpression produced noticeable effects on petiole and primary shoot fibre development, suggesting that PtaHB1 is involved in primary xylem development. We also obtained evidence indicating that expression of PtaHB1 affected the transcriptome by altering the accumulation of 48 distinct transcripts, many of which are predicted to be involved in growth and cell wall synthesis. Most of them were down-regulated, as was the case for several of the poplar HD-Zip III sequences. No visible physiological effect of over-expression was observed on PtaHB7 transgenic trees, suggesting that PtaHB1 and PtaHB7 likely have distinct roles in tree development, which is in agreement with the functions that have been assigned to close homologs in herbaceous plants.

Conclusions: This study provides an overview of HD-zip III genes related to woody plant development and identifies sequences putatively involved in secondary vascular growth in angiosperms and in gymnosperms. These gene sequences are candidate regulators of wood formation and could be a source of molecular markers for tree breeding related to wood properties.
\end{abstract}

\section{Background}

The differentiation of vascular tissues is an intensively studied aspect of plant development. Part of this interest is driven by the economic importance of xylem as a major constituent of forage crops, wood, and lignocellulosic biomass for transport fuels. Xylem is characterised

\footnotetext{
* Correspondence: john.mackay@sbf.ulaval.ca

'Département des Sciences du Bois et de la Forêt, Université Laval, 2405 rue de la Terrasse, Québec, QC, G1V OA6, Canada

Full list of author information is available at the end of the article
}

by highly specialised and easily identifiable water-conducting cell types, i.e. tracheids in gymnosperms and tracheary elements (TEs) in angiosperms. Xylem also contributes to the physical support of plant structures, which is imparted by either fibres (in angiosperms) or tracheids. Primary xylem arises through the differentiation of pro-vascular cells near the apical meristem and secondary xylem differentiates from fusiform initials in the cambial zone [1]. Environmental conditions and developmental state modulate xylem composition and

\section{() Biomed Central}


properties [2], as well as cell characteristics [3], through the action of growth regulators such as auxin, ethylene, and gibberellins, together with regulatory proteins such as transcription factors.

Insights into the regulatory components of xylem development, including transcriptional regulators, have been derived from functional analyses in the herbaceous model plants Arabidopsis thaliana (L.) Heynh., Zinnia elegans (Jacq.), and Oryza sativa (L.) [4,5]. HOMEODOMAIN LEUCINE ZIPPER CLASS III (HD-Zip III) proteins represent a group of transcription factors that have been extensively implicated in the regulation of primary and secondary vascular tissue pattern formation, as well as lateral organ and cambial polarity in herbaceous annual plants. It stands to reason that HD-Zip IIIs may also play key roles in secondary vascular growth and wood formation in perennials including shrubs and trees, but there is relatively little evidence to elucidate such a role, except for the report by Ko et al. (2006) [6].

There are several different classes of plant homeobox genes [7]. One of the major groups of these genes is HD-Zip, which is divided into classes I to IV. Both the DNA-binding Homeodomain (HD) and the basic leucine zipper domain (bZIP), the latter of which has protein dimerization properties [8], are conserved in all four classes. Members of the HD-Zip III and IV classes also share a steroidogenic, acute regulatory protein-related domain associated with the lipid-Transfer (START) domain [9]. In addition, class III HD-Zips have a characteristic C-terminal MEKHLA domain that shares significant similarity with the PAS domain, reported to dimerize with the AP2 domain of the transcription factor DRN/ESR-1 [10] involved in embryo patterning and auxin transport [11].

Five different HD-Zip III proteins have been functionally characterised by different approaches in A. thaliana. They include Revoluta (REV/IFL-1/AVB-1), Phabulosa (phb/AtHB-14), Phavoluta (phv/AthHB-9), Corona (cna/ $A t H B-15$ ) and AtHB-8. Arabidopsis REVOLUTA (rev) mutants have altered interfascicular fibre development and impaired auxin polar transport $[12,13]$. Overexpression of $R E V$ in Arabidopsis resulted in weakly radialized vascular bundles, and altered leaf, stem and carpel organ abaxial, adaxial pattern polarity. Overexpression of the $Z$. elegans $Z e H B-12$, a homologue of $R E V$, led to an increased number of xylem precursor cells and the accumulation of a variety of transcripts, including brassinosteroid-related sequences and vascular preferential transcripts in Zinnia [14]. Analyses of double phb:phv mutants showed that the two genes share redundant functions both in establishing organ polarity and in vascular development [15]. In Arabidopsis, $A t H B-8$ is an early marker for procambial development, vein patterning, and differentiation [16]. Its over- expression caused ectopic proliferation of xylem cells and precocious initiation of secondary growth; however, the $A t h b-8$ loss-of-function mutant had no obvious vascular phenotype [17]. In contrast, cha mutants and antisense plants have increased vascular tissues and defects in organ polarity [18], while CNA over-expression leads to smaller vascular bundles, indicating that it likely acts as a negative regulator of procambial cell identity or proliferation. Transcript accumulation in a few HD-Zip III sequences is regulated by auxin (specifically $A t H B-8$ ) [16] and brassinosteroids [12]. Post-transcriptional gene silencing by microRNAs is highly conserved in plants and specifically targets all of the HD-Zip III genes through the binding of mir165/166 [19].

Functional analyses of HD-Zip III genes in herbaceous plants, including A. thaliana and Z. elegans, have provided a useful template against which similar functions regulating secondary vascular growth can be investigated in woody plants (shrubs, trees) [20]. As genetic selection and breeding activities in trees are being expanded to include genetic mapping and molecular markers, candidate genes like HD-Zip III are considered as potential markers which could be associated with wood properties. In this context, the aim of this study was to characterise the HD-Zip III transcription factor family and assess potential involvement in vascular development of trees. Previous reports $[21,22]$ have provided indications that the number of HD-Zip III genes and gene family structure may vary between species, especially between angiosperms and gymnosperms. We evaluated and compared gene family structure in poplars (Populus spp.) and white spruce Picea glauca (Moench) Voss with that described for herbaceous annuals to clarify the evolutionary status of HD-zip III in these groups. Transcript profiles were examined across several tissues to assess their putative involvement in secondary vascular growth. In poplar, the accumulation of HD-Zip III gene transcripts was specifically examined in differentiating secondary xylem (2X) in relation to auxin transport, a key driver of tracheary element differentiation [23]. The putative roles of poplar genes PtaHB1 and PtaHB7from to distinct well characterised subclades with contrasted functions in crops were examined with respect to overexpression effects upon vascular differentiation and RNA transcript profiles.

\section{Results}

\section{Sequence analysis of HD-Zip III genes from conifer and hardwood trees}

Four putative full-length HD-Zip III coding sequences were isolated from P. glauca by EST data mining, RTPCR, and RACE cloning (Rapid Amplification cDNA End) with degenerate primers. Two class-IV sequences from $P$. abies have been previously reported and were 


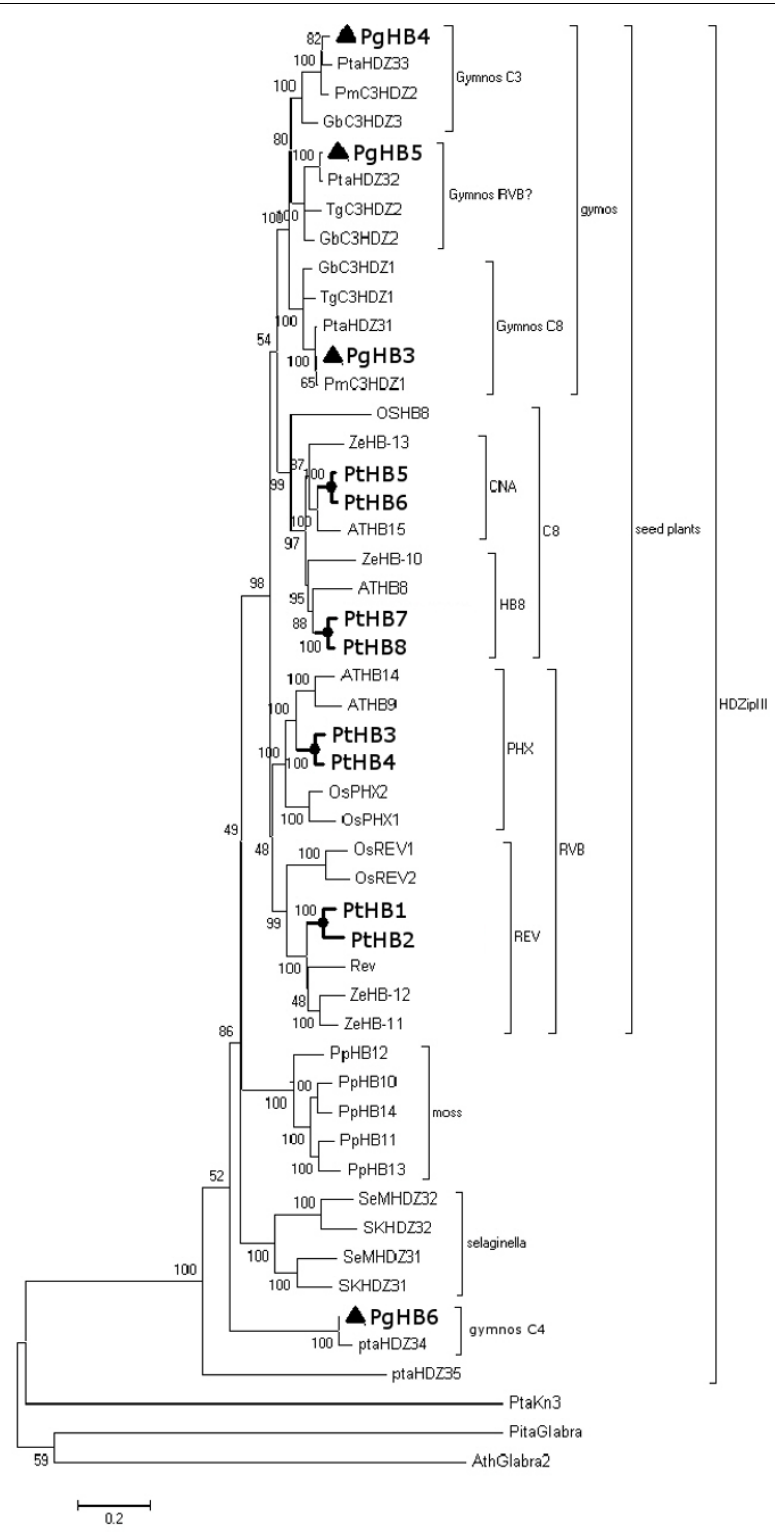

Figure 1 Cladogram showing the phylogenetic structure of the HD-Zip III gene family. The Neighbour-Joining (NJ) tree of HD-Zip III sequences was constructed from complete amino acid sequences using, with Poisson correction, 1000 bootstraps and pair-wise deletion parameters. Populus trichocarpa (PtHB1 to PtHB8: AY919616.1 to AY919623.1), Arabidopsis thaliana (Rev: AK229561.1, ATHB9: NM_102785.4, ATHB14: NM_129025.3, ATHB8: NM_119441.4, ATHB15: NM_104096.1), Physcomitrella patens (PpHB10 to PpHB14: DQ6567200.1 to DQ6567204.1), Picea glauca (HQ391914 to HQ391917), Pinus taeda (PtaHDZ31 to PtaHDZ35: DQ65720.1 to DQ65724.1), Zinnia elegans (ZeHB-10: AB084380.1, ZeHB-11:, ZeHB-12:, ZeHB-13:), Ginkgo biloba (GbC3HDZ1 ot GbC3HDZ3: DQ385525.1 to DQ385527.1), Taxus globosa (TgC3HDZ1: DQ385530.1, TgC3HDZ2: DQ385531.1), Pseudotsuga menziesii (PmC3HDZ1: DQ385528.1, PmC3HDZ2: DQ385529.1), Oryza sativa (OsHB8: AB374207.1, OsPHX1: AK103283, OsPHX2: AK103284, OsREV1: NM_001057934.1, OsREV2: AK100250.1), Selaginella kraussiana (SKHDZ31: DQ657196.1, SKHDZ32: DQ6571971), Selaginella moellendorffii (SeMHDZ31: DQ657198.1, SeMHDZ32: DQ657199.1). Black triangles are used for P. glauca sequences; bold characters are used for poplar.

denoted PaHB1 and PaHB2 [24]. Therefore, we designated the sequences that we isolated as $\mathrm{PgHB} 3$ [25] to PgHB6 (Additional file 1 Figure 1 HQ391914 to HQ391917). Predicted amino-acid sequences display the structural features of HD-Zip III, except that PgHB6 has a partially degenerated leucine zipper motif.
The Populus trichocarpa genome sequence [26] was reported to contain eight different HD-Zip III sequences, which are designated $H B 1$ to $H B 8$ [6]. HDZip III genes are distributed on seven of the nineteen poplar chromosomes (Additional file 1). We isolated full-length coding cDNA sequences for eight on the 
putative poplar HD-Zip III genes by RT-PCR, amplification, starting from the $P$. trichocarpa (Torr. \& Gray) $\times$ P. deltoides (W. Bartram) hybrid clone H11-11 and from the $P$. tremula Minch $\times P$. alba L. clone 717-1B4. For each of the eight cDNA clones, nearly perfect sequence identities were used to match the cDNA sequences with previously identified ESTs and genes predicted from the poplar genome [6], thus providing evidence that all of the predicted genes are expressed in Populus spp.

There are five HD-Zip III genes in the Arabidopsis genome belonging to the two major phylogenetic clades RVB and C8, each of which is divided into two subclades [27]. Floyd et al. (2006) [21] and Prigge and Clark (2006) [22] conducted phylogenetic investigations that included HD-zip III sequences from diverse plants, along with full-length and partial Pinus taeda L. cDNA sequences. They concluded that conifer HD-Zip III genes could be assigned to the two major angiosperm clades of C8 and RVB, but two of the conifer sequences were likely part of gymnosperm-specific clades. In this report, a neighbour-joining (NJ) tree [28] was constructed with complete amino acid sequences from several seed plants, including gymnosperms such as $P$. glauca and P. taeda, and angiosperms such as A. thaliana and $P$. trichocarpa, as well as lower plants such as the moss Physcomitrella patens (Hedw.) Bruch \& Schimp. The resulting tree topology was consistent with previous reports; however, our data suggest that conifer sequences may in fact be uniquely represented in the C8 clade and absent in the RVB clade (Figure 1). The conifers that we analysed may thus have three $\mathrm{C} 8 \mathrm{mem}$ bers, including sequences previously assigned to the RVB clade. The full-length $P$. glauca PgHB6 and the partial P. taeda PtaHD-34 and PtaHD-35 fell outside angiosperm clades and formed a monophyletic group, consistent with previous reports [21,22]. Sequence similarity and tree topology clearly grouped the Populus sequences as four pairs of closely related paralogues, which is consistent with the ancestral salicoid genomewide duplication and reorganisation described in modern Salicaceae [29].

\section{HD-zip III transcripts accumulate during secondary vascular growth in Picea and Populus}

Transcript accumulation was profiled in young $P$. glauca and $P$. trichocarpa $\times$ deltoïdes trees (refered as $P t d H B$ ) grown under controlled conditions by using RT-qPCR to compare steady mRNA levels in several organs and tissues (Figure 2). Transcripts of the four spruce sequences accumulated preferentially in the differentiating secondary xylem of stems (2X) and roots (R2X) and gave similar profiles overall (Figure 2A). PgHB3, PgHB4 and $P g H B 5$ RNAs were also abundant in the differentiating secondary phloem (2P), and PgHB5 had the highest relative abundance in the young foliage (YL) (Figure $2 \mathrm{~A})$. The data suggested that the different transcripts differ substantially in abundance since the normalised number of RNA molecules varied by two orders of magnitude between the highest and lowest RNAs, i.e., $P g H B 3$ and $P g H B 6$, respectively. The aforementioned data are consistent with putative roles in vascular differentiation, with little indication of diversification between the gene sequences.

Compared with spruce, poplar HD-Zip III genes gave more diversified transcript accumulation profiles across the panel of organs and tissues, even within pairs of closely related paralogues (Figure $2 \mathrm{~B}$ ). The pair PtdHB1 and PtdHB2, which are close homologues of REVO$L U T A$, gave relatively similar profiles across the panel, except that PtdHB1 was less abundant in mature and old leaves than in developing tissues. Furthermore, PtdHB1 transcript abundance was two orders of magnitude higher than PtdHB2. The pair PtdHB5 and PtdHB6, closest homologues of Corona/AtHB-15, shared similar transcript profiles which varied strongly between the organs surveyed. Both were clearly most abundant in the developing secondary xylem (2X), but also accumulated in the apex and primary stem. On average, PtdHB5 was five to ten times more abundant than PtdHB6. The pair PtdHB7 and PtdHB8, which are the closest homologues of $A t H B-8$, gave dissimilar and even opposite transcript profiles. PtdHB7 transcripts were abundant in nearly all organs and lowest in the apex (A) and developing secondary xylem (2X), whereas PtdHB8 transcripts were most abundant in these same tissues (A, 2X). Transcripts of PtdHB3, which was a close homologue of $P H V$ and $P H B$, largely accumulated in the apex and to a much lower degree than in other parts of the trees, especially the roots. Data are not reported for PtdHB4 because its amplification by RT-qPCR was not strong enough for reliable determinations.

\section{Over-expression of wild-type PtaHB1 and PtaHB7 genes in transgenic poplars}

Transgenic poplar trees that overexpressed the complete coding sequence of PtaHB1 and PtaHB7 were obtained to investigate the potential roles of these HD-Zip III genes in tree development. The hybrid poplar clone INRA-717-1B4 ( $P$. tremula $\times P$. alba) was transformed using Agrobacterium with either one of the PtaHB constructs or an empty vector control (WT). Several hygromycin-resistant and GUS-positive lines were recovered and used to produce viable plants grown to an average height of $1.20 \mathrm{~m}$ in the greenhouse. All of the lines had transgene transcript accumulation levels which were significantly above levels detected for the INRA-717 endogene (Table 1). Interestingly, all of the lines overexpressing PtaHB1 (UBI::PtaHB1) had a visible 
A)
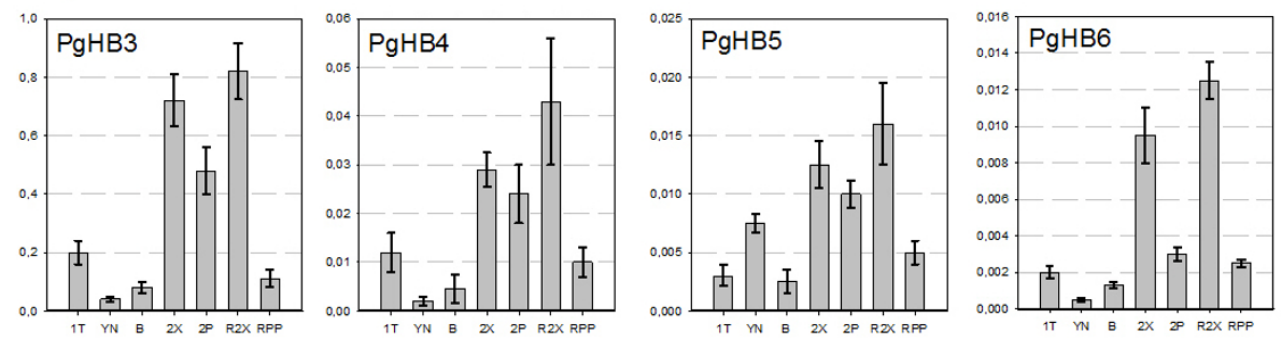

B)
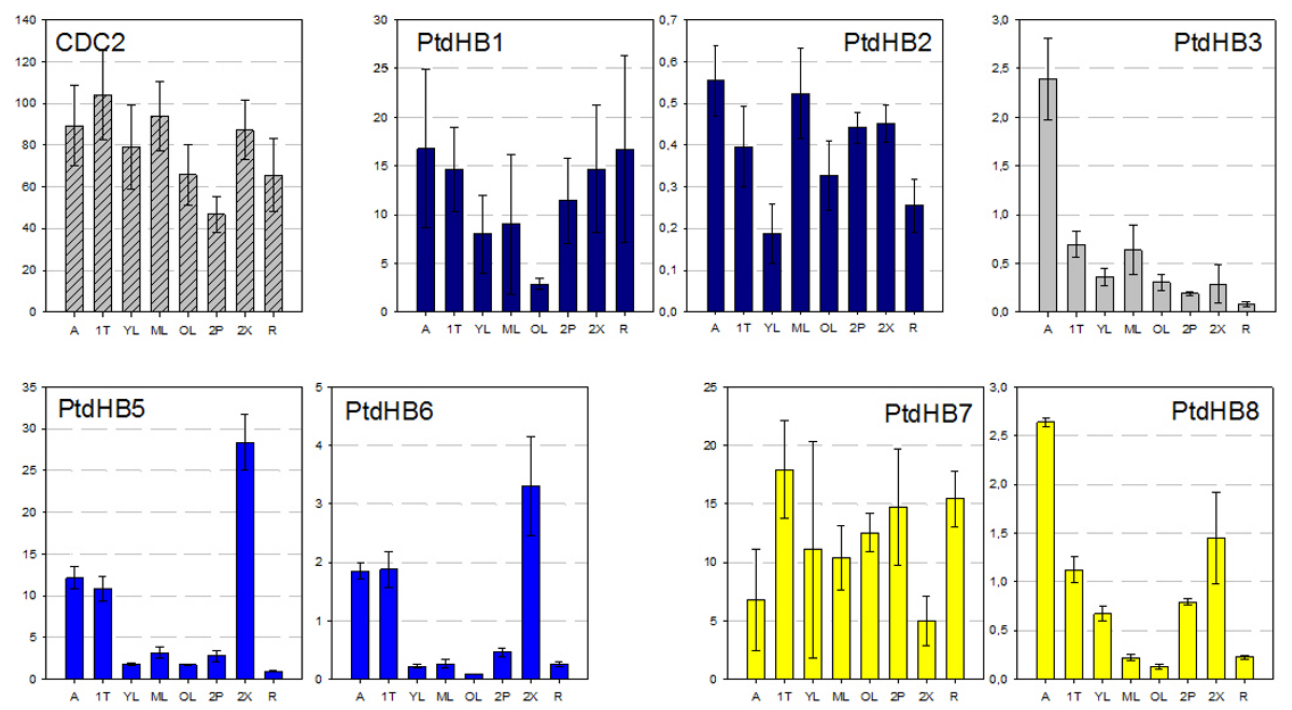

Figure 2 White spruce and poplar HD-Zip III transcript profiles across several organs and tissues. Steady-state RNA levels were determined by RT-qPCR with gene-specific primers. The Y-axis is the number of RNA molecules/ng total RNA (determined from a standard curve), which has been normalised based on the transcript accumulation level of a gene. A) Mean RNA level in P. glauca was analysed in duplicate in two independent biological replicates (one tree per replicate) \pm SD (error bar), and normalised based on the transcript accumulation levels of reference gene EF1a. B) Mean RNA level in $P$. trichocarpa $\times P$. deltoides (clone H11-11) from duplicate analyses of two biological replicates (two trees per replicate) $\pm S D$ (error bars), normalised with a $C D C_{2}$ reference gene. The recently duplicated poplar paralogues are colour-matched. The tissue codes (see Methods): shoot apex (A), portion of the main undergoing primary growth (1T), young needles from upper tree crowns (YN, in spruce); young leaves (YF, in poplar); mature leaves (MF); old leaves (OF); bark (B); stem secondary xylem (2X) and phloem (2P); root secondary xylem (R2X); phloem/phelloderm (RPP); and young root tips (R).

external phenotype that was not seen in the controls (Figure 3), but no phenotype was observed upon overexpression of PtaHB7 (data not show).

Further characterisation of the PtaHB1 transformed trees showed that PtaHB1 transgene transcripts were five to eight times more abundant than the PtaHB1 endogene in the controls. The most obvious phenotype in these trees was their drooping leaves. The trees appeared to have a water-stress phenotype (Figure 3A) which was clearly not the case given that they were grown alongside perfectly healthy control trees. Upon closer inspection, it was evident that PtaHB1 overexpression resulted in altered petiole development, causing the leaves to hang downward. Other than the petiole, the leaves seemed to develop normally and to be perfectly healthy, with no indications of altered water relations. On average, the transgenic poplars had petioles that were $15 \%$ shorter, and the angle between the adaxial side of the leaf and the stem was 30\% wider than those of control trees (Figure 3B). The increased angle and decreased length were statistically significant starting at the $10^{\text {th }}$ internode from the apex (where the first internode is the first leaf longer than $1 \mathrm{~cm})(p<$ 0.05) (Figure 3C, D).

The vascular organisation of petioles from mature leaves was examined to further investigate the altered development. Cell wall autofluorescence associated with lignin accumulation was observed in transverse sections under UV-illumination, and clearly indicated that the distribution of fibres and vessels was altered in the 
Table 1 Relative transcript abundance of HD-Zip III gene family members in transgenic poplars

\begin{tabular}{|c|c|c|c|c|}
\hline $\begin{array}{l}\text { Transgene } \\
\text { construct }\end{array}$ & Gene & $\begin{array}{c}\text { Mean } \log _{2} \\
\text { ratio }\end{array}$ & SD & $\begin{array}{c}p- \\
\text { value }\end{array}$ \\
\hline \multirow[t]{7}{*}{ UBI:PtaHB-1 } & $\begin{array}{l}\text { PtaHB- } \\
1^{* * *}\end{array}$ & 2.7320 & 0.4340 & $<0.001$ \\
\hline & PtaHB-2 & -0.7020 & 0.4580 & 0.0540 \\
\hline & PtaHB-3 & -0.5330 & 0.4730 & 0.1500 \\
\hline & PtaHB-5* & -0.7920 & 0.4960 & 0.0430 \\
\hline & PtaHB-6 & -0.5060 & 0.4840 & 0.1770 \\
\hline & PtaHB-7 & -0.4920 & 0.4490 & 0.1470 \\
\hline & PtaHB-8 & -0.5100 & 0.4600 & 0.1280 \\
\hline \multirow[t]{7}{*}{ UBI:PtaHB-7 } & PtaHB-1* & 0.5521 & 0.1869 & 0.0318 \\
\hline & PtaHB-2 & 0.4272 & 0.3613 & 0.2761 \\
\hline & PtaHB-3* & 0.7675 & 0.1940 & 0.0148 \\
\hline & PtaHB-5* & 0.7263 & 0.1578 & 0.0046 \\
\hline & PtaHB-6 & 0.6951 & 0.2727 & 0.0573 \\
\hline & $\begin{array}{l}\text { PtaHB- } \\
7^{* * *}\end{array}$ & 2.8634 & 0.2689 & 0.0008 \\
\hline & PtaHB-8 & 0.3194 & 0.1490 & 0.0838 \\
\hline
\end{tabular}

Total RNA from the same samples as those used for the microarray profiling: portion of the main undergoing primary growth (IT) for UBI::PtaHB1; scrapped secondary xylem (S2X) for UBI::PtaHB7 transgenic poplar. ${ }^{*} \mathrm{p}<0.05,{ }^{* * *} \mathrm{p}<$ 0.001 (Student's $t$ test, $N=6$ ); SD, one standard deviation.

transgenic trees (Figure 4A-B). The ratio of fibres (small lignified cells; Figure 4) to vascular elements (large lignified cells) was 0.80 in the transgenic trees, compared to 1.55 in the controls $(p<0.001)$.

Furthermore, quantitative determinations of fibre lengths in stems and petioles through FQA (Fiber Quantitative Analyser, Methods) showed that petiole fibres from immature (LPI 6) and mature leaves (LPI 21 and LPI 43) were slightly, but significantly shorter in the transgenic trees $(p<0.05$, Figure 4D). Shorter fibre classes $<0.5 \mathrm{~mm}$ were over-represented in the transgenic trees, whereas longer fibre classes (from 0.75 to 1 , from $2.0 \mathrm{~mm}$ and up) were significantly under-represented compared to the control trees $(p<0.05$, Figure $4 \mathrm{C})$. The fibre length classes from primary stems (internode between LPI 5 and LPI 6) followed a similar distribution pattern, but the mean fibre length was not significantly different between controls and transgenics. Secondary xylem fibres from the main stem, which were sampled from the same internodes as the petioles (LPI 6, LPI 21, LPI 43), did not differ between the transgenic and wild-type trees.

\section{Effect of PtaHB1 over-expression on the transcriptome}

Primary stem tissues from two control lines (two trees per line, $\mathrm{n}=4$ individual samples) and two PtaHB1 transgenic lines (two trees per line, $\mathrm{n}=4$ individual samples) were compared using a $3.4 \mathrm{~K}$ low redundancy

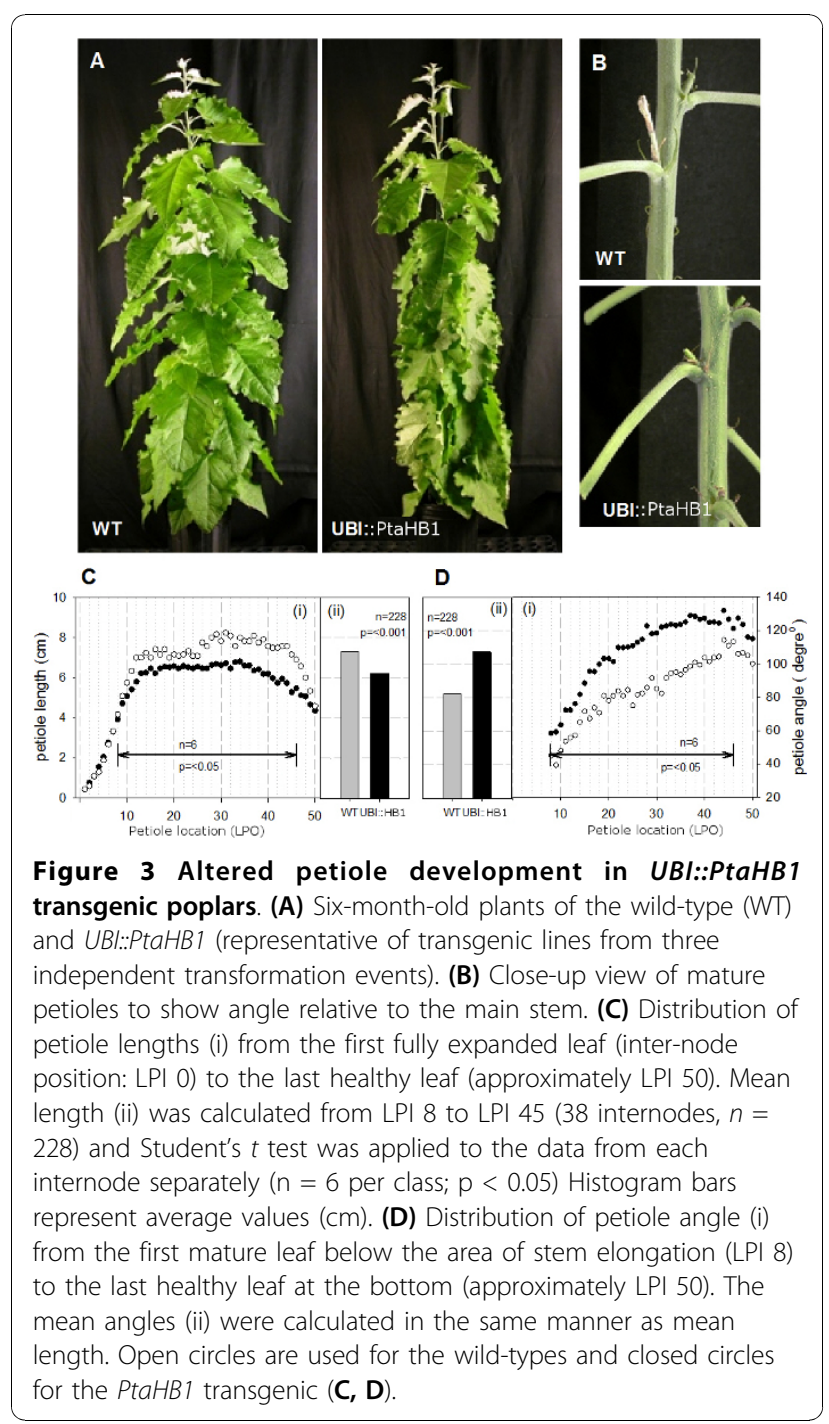

cDNA microarray (GSE24703 for raw data on GEO database). A total of 48 transcripts that accumulated differentially were expressed with a false discovery rate (FDR, [30]) threshold set to $5 \%(q<5.00)$ (SAM package release $3.0 ;$ [31]). Out of the 48 significantly misregulated genes, 8 transcripts were up-regulated and 40 were down-regulated in the transgenic trees (Table 1). The portion of the stem that we targeted in this analysis is also the part of the tree where petioles are actively developing and growing. Approximately one-third of the misregulated genes (14 out 48) had strong statistical support $(q<0.001)$. However, the fold-change of all genes identified was less than two $(-1<\mathrm{M}<1)$.

RT-qPCR analyses were carried out with gene-specific DNA primer pairs representing 20 putatively misregulated genes, to confirm the microarray data. These analyses used the same RNA samples as those used for microarray profiling plus two additional biological 

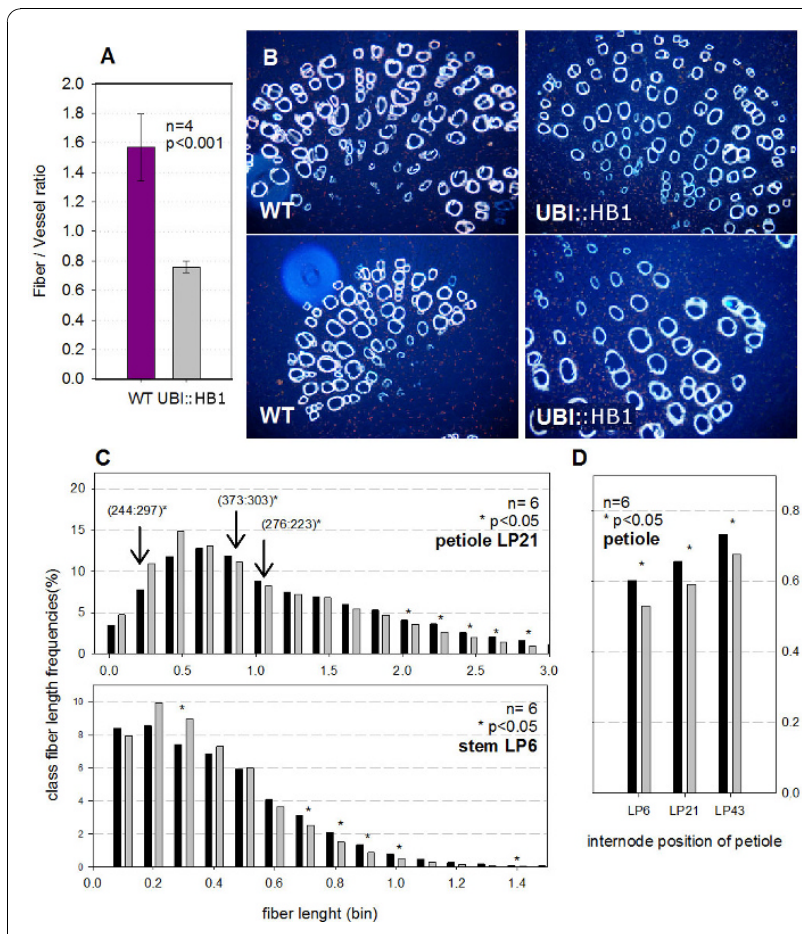

Figure 4 Altered fibre development in petioles and stems of transgenic UBI::PtaHB1 poplars. (A) Mean ( \pm SD) ratio of fibre vessel elements determined from image analyses based on four separate petioles for two transgenic lines carrying the UBI::HBI construct and one wild-type differed significantly according to Student's $t$ test $(p<0.001)$. (B) Cross-sections of mature petiole (40x, LP 21), observed under UV-illumination to reveal autofluorescence of lignified cell walls of fibre and vessel elements. (C) Distribution of fibre lengths in mature petioles (LPI 21) and partially lignified stems (LPI 6) determined by FQA from an average 8000 cells per sample. Each histogram bar represents the average proportion (\%) of cells in a given length class (or bin) from two transgenic lines (three plants each) and six wild-type plants. Numbers in brackets are the number of fibres counted for selected bins. (D) Average fibre length determinations at three stages of development (LPI 6, LPI 21, LPI 43). Bars indicate average length \pm

SD of 6 samples analyzed for each treatment. ${ }^{*}$ indicate fiber counts $(\mathrm{D}$, whole population or $\mathrm{C}$, bins) that differed significantly between transgenic and control trees (one-way ANOVA at $p<0.05$ ).

replicates $(n=6)$. Fold difference ratios from RT-qPCR results showed that twelve transcripts were congruent with the microarray results, while four genes gave no difference and four genes yielded conflicting results (Table 2). Data indicate that we were able to validate a subset of these misregulated sequences by RT-qPCR, which is consistent with a previous study reporting low rates of RTqPCR validation when microarray fold-changes are less than two [32]. Thus, our relatively low validation rate is not surprising and could be explained by other factors, including cross-hybridisation of closely related genes to the cDNA probes, for which we could not account [33].

The predicted functions of the misregulated transcripts in the PtaHB1 transgenics were examined and separated into four categories: growth factor-related, cell wall-related, membrane trafficking, and general functions. The growth factor group included sequences related to brassinosteroid action, which are putative leucine-rich BAK1-like proteins (CN520805, CN519565). These genes were down-regulated and suspected to be involved in steroid signal transduction [34]. Genes for ethylene perception and response were also down-regulated (HO702822, CN522424, and HO702885). Cell wall-related sequences were an abundant category of down-regulated transcripts. Other sequences related to cell expansion and cell proliferation were down-regulated, including sequences encoding two fasciclin-like proteins (CN518490) [35] two glyoxalases (CN519263, CN521180) [36], a farnesylated protein (HO702768) [37] and an elongation factor 2 (CN524724). The down-regulated sequences also included a 4CL gene (CN522696) that is involved in the synthesis of G-lignin precursors, and which is consistent with the decrease in auto-fluorescent fibres [38]. The up-regulated sequences encoded transketolase-like proteins putatively involved in isoprenoid biosynthesis (CN523609) [39] and in decreasing cell proliferation in preparation to dormancy [40].

The impact of PtaHB1 and PtaHB7 transgene expression on the other HD-Zip III gene transcripts was investigated in the transgenic poplars (Table 1). In general, the UBI::PtaHB1 constructs led to decreased transcript accumulation of all other HD-Zip III genes. However, the number of RNA molecules was quite variable and the effect was significant only for PtaHB5 (Student's t test, mean $\log _{2}$ ratio $\left.-0.7920, \mathrm{p}=0.0430\right)$. In UBI:: PtaHB7 transgenic trees, the HD-Zip III genes had slightly increased transcripts levels, but only PtaHB1, PtaHB3 and PtaHB5 were significantly upregulated (mean $\log _{2}$ ratio $0.5521, \mathrm{p}=0.0318 ; 0.7675, \mathrm{p}=0.0148$ and $0.7263, \mathrm{p}=0.0046)$.

\section{Accumulation of some, but not all HD-Zip III transcripts is linked to auxin in Poplar}

Given that some HD-Zip III genes have been linked to auxin transport during vascular development [12] and that PtaHB1 overexpression affected the accumulation of several transcripts related to growth regulators, we examined whether or not auxin influenced transcript accumulation of HD-Zip III genes in developing secondary xylem of young poplar trees. Removal of the stem apex, which is the primary source of auxin in the plant, significantly decreased the transcript level of PtdHB5 in the xylem tissue by nearly four-fold (mean $\log _{2}$ ratio $=-1.9739$ ) and had a similar effect PtdHB7 but it was not found to be statistically significant (mean $\log _{2}$ ratio $=-1.6421 ; \mathrm{p}$-value = 0.0776), and did not affect PtdHB1 (Table 3). The application of N-(1-naphthyl) phthalamic acid (NPA) to a portion of the stem undergoing secondary growth (see 
Table 2 Misregulated gene profiles from microarray analysis comparing transgenic UBI::PtaHB1 and wild-type lines and RT-qPCR validations

\begin{tabular}{|c|c|c|c|c|c|c|c|}
\hline \multirow[b]{2}{*}{$\begin{array}{l}\text { EST } \\
\text { (Genbank) }\end{array}$} & \multirow{2}{*}{$\begin{array}{l}\text { Poplar Gene ID. } \\
\text { Populus trichocarpa } \\
\text { Genome V } 2.0^{1}\end{array}$} & \multicolumn{2}{|r|}{ Functional Annotation } & \multicolumn{2}{|c|}{ Microarray results } & \multicolumn{2}{|c|}{ RT-qPCR validation } \\
\hline & & $\begin{array}{l}\mathrm{E} \\
\text { value }\end{array}$ & *POPTR BlastN **NCBI BlastX & $\begin{array}{l}\text { q- } \\
\text { value } \\
\text { (\%) }\end{array}$ & $\begin{array}{l}\text { M (log2 fold } \\
\text { difference) }\end{array}$ & $\begin{array}{l}\text { M (log2 fold } \\
\text { difference) }\end{array}$ & SD \\
\hline CN517570 & POPTR_0004s23850 & $\mathrm{E}-45$ & *predicted protein & 3.065 & -0.261 & $\mathrm{~N} / \mathrm{A}$ & \\
\hline CN517617 & POPTR_0006s12510 & 0 & ${ }^{* *}$ s-adenosylmethionine synthase 6 & 2.384 & 0.333 & -0.833 & 0.453 \\
\hline CN517648 & POPTR_0005s11070 & $\mathrm{E}-120$ & **Peroxidase (PO3) & 0.000 & -0.275 & 0.017 & 0.195 \\
\hline CN517711 & POPTR_0004s24390 & E-90 & *predicted protein & 3.065 & -0.213 & N/A & \\
\hline CN517879 & POPTR_0001s28710 & $\mathrm{E}-42$ & **Serine/threonine protein kinase & 0.000 & -0.488 & $-0.090 *$ & 0.693 \\
\hline CN518033 & POPTR_0010s14250 & 0 & ${ }^{*}$ predicted protein & 3.065 & -0.364 & N/A & \\
\hline CN518196 & scaffold_6:22787285..22789257 & $\mathrm{E}-05$ & ${ }^{* *}$ protein kinase & 2.258 & -0.179 & N/A & \\
\hline CN518487 & POPTR_0010s12680 & $\mathrm{E}-91$ & $\begin{array}{l}\text { **mitochondrial beta subunit of F1 ATP synthase } \\
\text { (PtrAtpB) }\end{array}$ & 2.384 & 0.320 & N/A & \\
\hline CN518490 & POPTR_0004s22020 & $\mathrm{E}-53$ & **Fasciclin-like AGP 10 & 3.065 & -0.545 & $-0.678 *$ & 0.365 \\
\hline CN518917 & POPTR_0010s05180 & $\mathrm{E}-27$ & ${ }^{* *}$ putative polygalacturonase, pectidase & 2.600 & -0.200 & N/A & \\
\hline CN518924 & POPTR_0015s00430 & $\mathrm{E}-41$ & **Plastid-specific $30 \mathrm{~S}$ ribosomal protein 1 & 3.575 & -0.293 & N/A & \\
\hline CN518966 & POPTR_0003s13440 & $\mathrm{E}-73$ & ${ }^{* *}$ progesterone 5 -beta-reductase- $\mathrm{A}$ & 2.384 & 0.323 & N/A & \\
\hline CN519065 & POPTR_0008s14360 & $\mathrm{E}-171$ & *Myoinositol oxygenase, Aldehyde reductase & 0.000 & 0.449 & $0.323 *$ & 0.838 \\
\hline CN519230 & POPTR_0015s03670 & 0 & ${ }^{*}$ predicted protein & 2.258 & -0.272 & $-0.044 *$ & 0.837 \\
\hline CN519263 & POPTR_0022s00750 & $\mathrm{E}-32$ & **Lactoylglutathione lyase, Glyoxalase putative & 3.651 & -0.204 & N/A & \\
\hline CN519295 & POPTR_0012s14780 & $\mathrm{E}-30$ & ${ }^{* *}$ Coatomer delta subunit & 3.065 & -0.266 & N/A & \\
\hline CN519368 & POPTR_0004s21650 & 0 & *phosphate-responsive 1 family protein & 0.000 & 0.567 & -0.246 & 0.404 \\
\hline CN519565 & POPTR_0001s22700 & $\mathrm{E}-55$ & $\begin{array}{l}\text { **leucine rich protein, Brassinosteroid insensitve } \\
\text { 1-associated receptor kinase (BAK-1) }\end{array}$ & 2.768 & -0.314 & $-0.710 *$ & 0.326 \\
\hline CN520095 & POPTR_0005s22210 & $\mathrm{E}-108$ & ${ }^{* *}$ Oxidoreductase activity protein & 0.000 & 0.390 & -0.803 & 0.446 \\
\hline CN520368 & POPTR_0008s06940 & $\mathrm{E}-41$ & ${ }^{* *}$ Cys-3-His zinc finger protein & 4.086 & -0.332 & $-0.044 *$ & 0.619 \\
\hline CN520805 & POPTR_0009s02400 & $\mathrm{E}-93$ & $\begin{array}{l}\text { *leucine rich protein }{ }^{* *} \text { Brassinosteroid insensitve } \\
1 \text {-associated kinase repector, (BAK-1) }\end{array}$ & 0.000 & -0.203 & $-0.424 *$ & 0.344 \\
\hline CN521180 & POPTR_0001s13540 & $\mathrm{E}-50$ & $\begin{array}{l}\text { **Lactoylglutathione lyase/glyoxalase } 1 \text { family } \\
\text { protein }\end{array}$ & 0.000 & -0.281 & $-0.287 *$ & 0.432 \\
\hline CN521321 & POPTR_0007s10200 & $\mathrm{E}-14$ & **hydrolase, alpha/beta fold family protein & 2.200 & -0.227 & N/A & \\
\hline CN521367 & POPTR_0006s29050 & $\mathrm{E}-27$ & **ABC1 family protein & 3.065 & -0.255 & N/A & \\
\hline CN521610 & POPTR_0009s12310 & $\mathrm{E}-86$ & *Predicted protein & 4.086 & -0.520 & 0.059 & 0.591 \\
\hline CN521704 & POPTR_0010s24930 & $\mathrm{E}-21$ & **DnaJ homolog & 3.651 & -0.233 & N/A & \\
\hline CN521866 & POPTR_0011s04190 & $\mathrm{E}-49$ & **Armadillo/beta-catenin repeat family protein & 0.000 & -0.243 & N/A & \\
\hline CN522073 & POPTR_0017s06630 & $\mathrm{E}-29$ & **EXS family protein & 0.000 & -0.259 & N/A & \\
\hline CN522222 & POPTR_0009s10300 & $\mathrm{E}-142$ & $\begin{array}{l}{ }^{*} \mathrm{C} 3 \mathrm{HC} 4 \text { ring } \mathrm{Zn} \text {-finger, Anaphase-promoting } \\
\text { complex (APC), subunit } 11\end{array}$ & 3.065 & -0.258 & N/A & \\
\hline CN522424 & POPTR_0008s23260 & E-109 & ${ }^{*}$ Ethylene response factor (ERF35) Pt-RAP2.4 & 2.768 & -0.358 & N/A & \\
\hline CN522566 & POPTR_0005s12510 & E-163 & *Unknown function & 0.000 & -0.407 & N/A & \\
\hline CN522696 & POPTR_0012s09650 & $\mathrm{E}-81$ & ${ }^{* *} 4$-coumarate-coa ligase (Ptr4CL9) & 2.768 & -0.214 & N/A & \\
\hline CN522933 & POPTR_0005s19400 & $E-21$ & $\begin{array}{l}\text { **Banched-chain amino acid aminotransferase, } \\
\text { putative }\end{array}$ & 0.000 & -0.209 & N/A & \\
\hline CN522970 & POPTR_0005s27930 & $\mathrm{E}-14$ & **bZIP transcription factor family protein & 3.651 & -0.297 & $-0.237 *$ & 0.379 \\
\hline CN523006 & POPTR_0006s19770 & $\mathrm{E}-48$ & **phytocyanin-like arabinogalactan-protein & 0.000 & 0.613 & -0.890 & 0.762 \\
\hline CN523531 & POPTR_0006s23940 & E-104 & $\begin{array}{l}\text { **phytanoyl-CoA hydroxylase (PhyH) } \\
\text { glycoproteins AGP19 }\end{array}$ & 3.065 & -0.299 & $-0.330 *$ & 0.484 \\
\hline CN521717 & POPTR_0002s14730 & $\mathrm{E}-26$ & **Transketolase & 0.000 & 0.261 & N/A & \\
\hline CN523609 & POPTR_0011s13810 & $\mathrm{E}-126$ & *Translation initiation factor activity SUI1 & 3.065 & -0.281 & $-0.192 *$ & 0.560 \\
\hline CN522357 & POPTR_0007s08390 & $E-136$ & **Elongation factor EF-2 & 3.065 & -0.336 & $-0.533 *$ & 0.427 \\
\hline
\end{tabular}


Table 2 Misregulated gene profiles from microarray analysis comparing transgenic UBI::PtaHB1 and wild-type lines and RT-qPCR validations (Continued)

\begin{tabular}{|c|c|c|c|c|c|c|c|}
\hline HO702741 & POPTR_0007s12770 & 0 & *Unknown function & 0.000 & -0.397 & $-0.679 *$ & 0.300 \\
\hline HO702768 & POPTR_0009s13750 & $\mathrm{E}-28$ & ${ }^{* *}$ Farnesylated protein & 2.145 & -0.388 & N/A & \\
\hline HO702822 & POPTR_0010s00900 & $E-37$ & $\begin{array}{l}{ }^{*} \mathrm{AP} 2 / \text { Ethylene response factor domain- } \\
\text { containing transcription facctor }\end{array}$ & 3.651 & -0.517 & -1.034 & 0.860 \\
\hline HO702830 & POPTR_0010s01590 & $E-160$ & *Late embryogenesis abundant protein 3 & 3.065 & -0.868 & $-0.983 *$ & 0.561 \\
\hline HO702837 & POPTR_0015s06030 & $\mathrm{E}-117$ & *Unknown fonction & 3.065 & -0.234 & N/A & \\
\hline HO702874 & POPTR_0010s11840 & E-66 & *DUF26 & 2.600 & -0.240 & N/A & \\
\hline HO702885 & POPTR_0002s20260 & E-61 & ${ }^{* *}$ Ethylene receptor 1 (ETR1) & 3.651 & -0.280 & $\mathrm{~N} / \mathrm{A}$ & \\
\hline HO702895 & scaffold_20:856315..856454 & $E-45$ & *Unknown function & 0.000 & -0.360 & N/A & \\
\hline HO703041 & POPTR_0008s20950 & $E-128$ & $\begin{array}{l}\text { *DUF588, Nitrate, Iron, Fromate dehydrogenase, } \\
\text { integral membrane protein }\end{array}$ & 3.065 & -0.914 & N/A & \\
\hline
\end{tabular}

\footnotetext{
Sequence identified from poplar genome V1.1; was not found in V2.0

${ }^{2}$ Microarray results validated by RT-qPCR are highlighted in black, while grey highlights indicate results were not validated by RT-qPCR. N/A not assayed. Microarray results are for primary stem tissues. Fold differences $(M)$ were derived from two independent transgenic lines (two plants per line) (see methods, raw data available on NCBI GEO database, accession \# GSE24703).
}

Methods) significantly decreased transcript abundance for PtdHB1, PtdHB5 and PtdHB8 (mean $\log _{2}$ ratios of $-1.2010,-2.0375,-0.7031$, respectively). The only gene affected by both treatments was PtdHB5.

\section{Discussion}

Vascular development is a finely tuned process that is integral to primary growth, i.e., stem elongation, as well as secondary growth, i.e., radial or diameter growth. The differentiation and growth of the primary vasculature

Table 3 Differential transcript level of HD-Zip III genes in developing secondary xylem from $P$. trichocarpa $\times P$. deltoides (clone H-1111) following removal of the apex or application of an auxin transport inhibitor (NPA), compared to untreated controls

\begin{tabular}{ccccc}
\hline Gene & Treatment & Log $_{\mathbf{2}}$-fold difference & SD $^{\mathbf{1}}$ & $\boldsymbol{p}_{\text {-value }} \mathbf{v}^{\mathbf{2}}$ \\
\hline PtdHB1 & apex (-) & -0.5062 & 1.17 & 0.1092 \\
& NPA & -1.201 & 1.09 & $0.0270^{*}$ \\
\hline PtdHB2 & apex (-) & 0.4671 & 1.17 & 0.4777 \\
& NPA & -0.4111 & 0.89 & 0.4307 \\
\hline PtdHB3 & apex (-) & 0.1637 & 0.91 & 0.4663 \\
& NPA & -0.4473 & 0.71 & 0.4762 \\
\hline PtdHB5 & apex (-) & -1.9739 & 0.69 & $0.0461^{*}$ \\
& NPA & -2.0375 & 1.22 & $0.0496^{*}$ \\
\hline PtdHB6 & apex (-) & -0.9145 & 0.91 & 0.1346 \\
& NPA & -1.4915 & 1.14 & 0.1090 \\
\hline PtdHB7 & apex (-) & -1.6421 & 0.76 & 0.0776 \\
& NPA & -1.3204 & 1.26 & 0.1725 \\
\hline PtdHB8 & apex (-) & -0.5686 & 0.98 & 0.1488 \\
& NPA & -0.7031 & 1.93 & $0.0455^{*}$ \\
\hline
\end{tabular}

${ }^{1} \mathrm{SD}$ is one standard deviation.

${ }^{2} \mathrm{p}$-value is based on Student's $t$ test, $\mathrm{N}=2$ pools of 3 samples); ${ }^{*}$ indicates the treatment had significant effect a threshold of 0.05 derives from the apical meristem, whereas secondary vascular tissues derive from the cambium. The specific spatio-temporal control and action of regulators enable the coordinated differentiation of the vasculature and other tissues during plant development. In plant model systems such as Arabidopsis and Zinnia, it has been established that key events underlying vascular differentiation involve a few different HD-Zip III transcription factors. This small family of regulators are known for their overlapping expression profiles and their functional redundancy. The aim of this study was to develop insights into the role of HD-Zip III genes in secondary xylem formation in forest trees. We examined the HDZip III gene family in two unrelated tree species belonging to the angiosperms (Populus spp.) and the gymnosperms (P. glauca).

\section{Distinct HD-Zip III gene family evolution in gymnosperm} and angiosperm trees

Gene sequences isolated from the moss $P$. patens with features typical of HD-Zip genes of class I, II, and III clearly indicate that they were acquired early in plant evolution [41]. The sequence analyses presented here (Figure 1) are consistent with the idea that HD-Zips have evolved through gene or genome duplications and potential gene losses [21,22].

The phylogenetic tree we described (Figure 1) included four full length HD-Zip III cDNA sequences of P. glauca and was similar but not entirely congruent with the tree topology previously predicted with a Bayesian procedure that used full length and partial cDNA sequences from $P$. taeda $[21,22]$. On one hand, previous authors have reported that gymnosperm HD-Zip III sequences could be assigned to both the C8 and RVB clades defined in angiosperm plants. On the other hand, 
they showed that gymnosperms also formed two independent clades not represented in angiosperms. Our results are consistent with the existence of gymnosperm clades with representatives from Pinus and Picea. These findings support the hypothesis that modern HD-Zip III family structure derives from four ancestral sequences, and that two of the ancestral sequences have been lost in angiosperms leading to clades C8 and RVB, whereas all four clades have potentially been retained in gymnosperms. However, our finding that the RVB clade lacked conifer sequences and the lack of a reference gymnosperm genome sequence led us to conclude that further analyses are needed to confirm whether or not gymnosperms are in fact represented in the RVB subclade.

Poplars have three more HD-Zip III sequences than Arabidopsis, which is consistent with the inferred genome evolution of the former [26]. The poplar sequences clearly formed four pairs of closely related paralogues. The salicoid plant lineage that gave rise to the family Salicaceae (including Populus spp.) appears to have undergone a relatively recent genome-wide duplication and reorganisation [26], whereas Arabidopsis is thought to have undergone genome size reduction [42]. These different evolutionary paths could have led to the loss of certain functions as well as neofunctionalisation or subfunctionalisation within the angiosperms.

\section{Transcription profiles identify HD-Zip III putatively involved in vascular development}

Delineating the potential role of HD-Zip III genes in regard with vascular development is aided by comparing RNA transcript accumulation in different organs, tissues and cell types, despite the overlapping profiles that may be observed within the family. Members of the C8 clade have been most strongly linked to vascular development and have not been implicated in leaf formation as such. In Arabidopsis, AtHB-15/CNA is expressed in procambial cells where it is involved in early initiation of vascular cells, and has been implicated in embryo polarity. The AtHB- 8 gene product has been shown to promote the proliferation and differentiation of xylem cells. Its expression also localizes to pro-cambium cells, in addition to being modulated by auxin [16]. The transcripts corresponding to the three $P$. glauca sequences we assigned to the $\mathrm{C} 8$ clade were detected in all tissues but preferentially in differentiating secondary vascular tissues both in the stem and in the roots. This observation may represent evidence in support of the phylogenetic position of Picea sequences $P g H B-3$ to $P g H B-5$, along with several other gymnosperm sequences, in clade $C 8$ rather than RVB. In Populus, there are four C8 sequences PtdHB5 to PtdHB8 with varied transcript accumulation profiles in vascular tissues. The accumulation of PtdHB5 and PtdHB-6 transcripts were also clearly preferential to secondary xylem tissues. In contrast, the paralogous sequences PtdHB7 and PtdHB8 have very dissimilar profiles and were distinctly not preferential to secondary xylem. These transcript accumulation profiles of PtdHB7 and PtdHB8 indicated that Populus $\mathrm{C} 8$ sequences may have undergone relatively recent neofunctionnalisation or subfonctionnalisation, compared to the pair of PtdHB5 and PtdHB6 which share the most similar expression patterns. Overall, it appears that gene duplications found in gymnosperm C8 clade, and even the more ancient duplications at the family level (PgHB6), have not led to strong diversification of expression profiles compared to that observed in angiosperms.

Lateral organ formation has been assigned to RVB clade that includes REV, AtHB-9 (PHB) and AtHB-14 $(P H V)$. The closely related genes $P H B$ and $P H V$ are involved in leaf polarity, while $R E V$ has been implicated in several developmental processes, including vascular cambium identity and activity, as well as fibre differentiation. Two putative homologues of Arabidopsis REV genes have been detected in the genomes of Populus, $Z$. elegans, $O$. sativa and $Z$. mays L. $[43,44]$. The functions of the Zinnia REV homologues appear to have diverged, with one being implicated in vascular development and the other in lateral organ formation [9]. In contrast, the Populus HB1 and HB2 have similar transcript patterns, except that $H B 2$ transcripts accumulate more strongly in maturing leaves (Figure 2B). Arabidopsis may represent a unique case with a $R E V$ paralog potentially having been lost during ancestral genomic rearrangements [42], and resulting in a gain of function for the remaining $R E V$ sequence in developing xylem and leaves. Ko et al. (2006) [6] found that PtdHB1 was associated with secondary growth in poplar stems and hypothesised that HD-Zip III genes played a role in secondary xylem differentiation in trees. Our expression survey indicated that PtdHB1 transcripts are present at a similar level in the apex, primary stems, secondary xylem, and young roots.

\section{Poplar HD-zip III genes play a role in fibre development}

Constitutive over-expression of the poplar PtaHB1 gene in poplar led to greater transcript abundance corresponding to this gene, and resulted in shorter petioles and a wider angle between the stem and adaxial side of the petiole. The fibres with reduced lignification and shorter length suggested that development of primary xylem fibres was either impaired or delayed in the transgenic trees. Our hypothesis is that the increased angle between the petioles and the stem is caused by a delayed or incomplete fibre development relative to leaf expansion. Asynchronous development may cause the petioles to lack the necessary strength to support a fully expanded leaf. This phenotype bears a resemblance to 
that of the ifl-1 mutant (REV gene) in which interfascicular fibre development is impaired and inflorescence stems lack sturdiness [43]. Moreover, it has been observed that $R E V$ gain-of-function promotes xylem differentiation and accumulation, leading to dysfunctional vascular patterning [13]. It thus appears that the PtaHB1 over-expression phenotype is closer to the ifl-1 mutant phenotype than the $R E V$ gain-of-function phenotype. This result contrasts with our initial hypothesis that PtaHB1 over-expression might promote fibre differentiation.

Furthermore, the vascular phenotype observed in PtaHB1 transgenic poplars was not consistent with previous observations that over-expression of non-mutated HD-Zip III genes had no effect on plant development because of gene silencing by microRNAs. In Arabidopsis, all of the HD-Zip III family transcripts are targeted and negatively regulated by microRNAs (MiR165/166) found in multiple copies in the genome [6]. Moreover, the over-expression of the HD-Zip III sequences was shown to trigger the production of these microRNAs [45]. An hypothesis to explain our observations may be that HD-Zip III PtaHB1 over-expression triggered the accumulation of microRNAs that down-regulated the other members of the HD-Zip III family. Transcript accumulation of the other poplar HD-Zip III sequences provided evidence in support of this hypothesis. Interestingly, PtaHB7 over-expression did not appear to have a similar effect on other family members.

It has long been known that fibre differentiation or stem elongation is controlled by growth factors such as auxin, GA and brassinosteroids, and that overproduction of auxin decreases primary stem elongation in Arabidopsis [46]. The REV/IFL-1 gene of Arabidopsis has been implicated in polar transport of auxin [43]. Considering the known relationship between plant growth regulators and HD-Zip III genes, the delay in petiole elongation in PtaHB1 transgenics may be linked to a perturbation of growth regulation activity. For example, auxin accumulation may be shifted due to altered polar transport. Our observations in young wild-type poplar trees suggested a putative link between auxin and the expression of three different HD-Zip III genes (PtdHB1, PtdHB5 and PtdHB8), the transcript levels of which were affected by the application of an auxin transport inhibitor (Table 3). This observation is consistent with the fact that those gene transcripts are well represented in secondary xylem (Figure 2B). Plant decapitation (from the apex to LPI 3 inclusively) significantly down-regulated transcripts of PtdHB5 and had a smaller effect on PtdHB7 transcripts. These results are partially consistent with previous reports from Arabidopsis, where AtHB-8 was clearly modulated by auxin. Gene regulation may have evolved differently in poplar compared to
Arabidopsis, reflecting the developmental and physiological differences between a woody perennial plant and an herbaceous annual.

The differentially expressed sequences identified in the PtaHB1 over-expressing poplars were classified into a few broad categories based upon putative functional assignments. Most of the transcripts were down-regulated and included sequences related to development pathways: brassinosteroid and ethylene growth regulators, ethylene perception and response, and putative steroid signal transduction proteins, in addition to cell wall-related and cell expansion or cell proliferation proteins. Many of the sequences have putative functions that appear consistent with developmental activities of IFL1/REV in Arabidopsis. They also appear to be consistent with the functions or pathways related to the phenotype observed in transgenic poplars.

\section{Conclusions}

The analysis of HD-Zip III genes in vascular development has been complicated by the complex interactions between family members and the pleiotropic nature of the mutant phenotypes [22]. Nevertheless, the experiments and findings reported here contribute to confirming the involvement of this group of genes in woody plants, including primary and secondary vascular development. Taken together, the observations indicate that HD-Zip III gene family structure has considerably diverged between angiosperms and gymnosperms, and suggest that individual genes within each taxonomic group have acquired distinct and specialised functions, some of which are related to secondary xylem growth. The phenotype observed upon over-expression of a wild-type poplar PtaHB1 gene represents a departure from previous reports in Arabidopsis, where gene silencing by miRNAs suppressed potential effects of overexpression. The transgenic poplars likely represent a useful system to continue investigating the functions of PtaHB1. Transcript profiling identified a set of sequences which may be targets of PtaHB1 or perhaps lay downstream of PtaHB1 in the developmental cascade. Microarray profiling experiments using whole-genome arrays and targeting of other plant tissues related to the transgenic phenotypes would likely help to consolidate the list of candidate targets.

Future studies could continue to explore and compare more broadly the role of HD-Zip III genes in primary and secondary vascular growth of woody plants, particularly the poplar PopHB5 (closest homologues to CNA) which appeared the most specific to secondary xylem of all other poplar HD-Zip III. In this regard, HD-Zip III knock-downs (RNAi or anti-sense) could potentially help to clarify the putative role of poplar HD-Zip III genes. Genes encoding regulators of wood formation 
potentially represent a rich source for identifying genetic markers of wood properties that could used in targeted breeding and selection. It typically takes $10-15$ years or more to grow trees to the point where they can be selected to develop improved or new varieties; therefore, if such markers are proven effective, they could potentially reduce the selection time and cost.

\section{Methods}

\section{Plant material and growth conditions}

Picea glauca (Moench) Voss (white spruce) tissues were obtained from two sources. The gene isolation work used plantlets of clone Pg-653, which are produced by somatic propagation by the Canadian Forestry Service (Klimaszewska et al., 2001). For the transcript-level survey, samples were obtained from two wild field grown 33-year-old trees in a progeny trial that had been established near Québec City. Two hybrid poplar clones were used: Populus trichocarpa (Torr. \& Gray) $\times P$. deltoides (clone H11-11, for gene expression experiments) and Populus tremula L. $\times$ P. alba L. clone INRA clone 717 1-B4 (referred to as clone 717 for transgenic analysis). Rooted softwood poplar cuttings were produced in 25 $\mathrm{cm}^{3}$ pots protected in clear plastic bags, transferred to 3 $\mathrm{L}$ pots after five weeks, and maintained in a greenhouse. Both the spruce and poplar plants were grown in a greenhouse with 16 hours light per day, with a temperature regime of $22 / 17^{\circ} \mathrm{C}$ (day/night), and relative humidity of at least $70 \%$. Natural daylight was supplemented with light from HQI-TS 400W/DH metal halogen lamps (Osram, Munich, Germany). Plants were fertilised weekly with $1 \mathrm{~g} \mathrm{~L}^{-1}$ 20/20/20 (N-P-K) and supplemented with calcium every two weeks.

\section{Tissues for transcript profile survey}

All tissues were frozen in liquid $\mathrm{N}_{2}$ immediately upon removal from the tree and stored at $-80^{\circ} \mathrm{C}$ until further use. Several organs/tissues were isolated from two 33year-old field-grown white spruce $P$. glauca for transcript accumulation profiling. These included the terminal leader $(1 \mathrm{~T})$, young needles from the upper crown $(\mathrm{YN})$, differentiated secondary phloem (2P) and xylem (2X), as well as bark (B) tissues collected from three 30$40 \mathrm{~cm}$ bolts taken from the lower third of the main stem. The secondary phloem and xylem issues were scraped with scalpel immediately after removing the bark: $2 \mathrm{P}$ was scrapped from the exposed inner side of the bark and the $2 \mathrm{X}$ from surface of the exposed wood. This method relies on cleavage of tissues at the cambial zone and yields relatively pure tissue types, although the purity of $2 \mathrm{X}$ and $2 \mathrm{P}$ samples were not verified as such. Similarly, tissues from large roots, including differentiating xylem (R2X) and phloem (RPP; with phelloderm) were collected taken in a one-meter radius from the base of the stem. Each sample was kept separate for total RNA extraction. Tissues were also collected from two trees of clone H11-11 selected to be similar in size, i.e., average of $80 \mathrm{~cm}$ tall and possessing at least 25 leaves with a plastochron index (LPI) greater than zero [47]. LPI 0 denotes a leaf blade that is $1 \mathrm{~cm}$ long and is undergoing laminar expansion. The tissues consisted of: Apex LPI 0 without leaves (A); shoot tips up to and including LPI 1 (1T); young leaves up to LPI 3 (YF); mature leaves LPI 15-16 (ML); old leaves LPI 30-31 (OL), and differentiating xylem and phloem scrapped with a scalpel from the portion of the main stems exhibiting secondary growth (2X and $2 \mathrm{P}$, respectively) between LPI 15 and LPI 20; and actively growing white coloured roots $(\mathrm{R})$.

\section{Auxin-related treatments in poplar}

Two treatments were applied to young poplar trees $(P$. trichocarpa $\times P$. deltoides H11-11, described above) averaging $80 \mathrm{~cm}$ in height in order to assess the potential effect of auxin on HD-Zip III transcript accumulation in poplar. Six randomly selected trees which possessed at least 25 leaves with a plastochron index (LPI) greater than zero were assigned to each treatment and to the control untreated group. The first treatment removed the shoot apex, which involved cutting off the top part of plants down to LPI 3 inclusively. The second treatment applied an inhibitor of polar auxin transport, $\mathrm{N}$-(1-naphthyl) phthalamic acid (NPA, $1 \mathrm{mM}$ for treatment and $0 \mathrm{mM}$ for control) mixed with lanolin as a carrier. The mixture of NPA and lanolin was placed on the bark (after removing the layer of cuticle), entirely covering a segment of the stem of $2.5 \mathrm{~cm}$ centered at the internode LP15, and covered with paraffin film. Secondary xylem tissues were collected $72 \mathrm{~h}$ after the treatments were applied, from internodes LPI14 to 16 and consisted of whole stem segments without the bark. For the NPA treated trees, the sampling consisted of stems segments without bark $3 \mathrm{~cm}$ above and $3 \mathrm{~cm}$ below the region of NPA application (LPI15 was excluded). The transcript accumulation data (see below) obtained from these tissues were analysed using Student's t test applied to each gene separately comparing the treated and untreated plants. For the NPA treated trees, tissues obtained below and above the region of application were analysed separately and the data were averaged for each tree.

\section{RNA extraction, sequence isolation and phylogenetic analyses}

Total RNA extractions were carried out using the CTAB method of Chang et al. (1993) [48] from spruce and poplar tissues, which were stored at $-80^{\circ} \mathrm{C}$ until used. The quantity and integrity of total RNA were evaluated 
by spectrophotometry (OD 260/280 ratio of $1.8: 1$ to 2.1:1), and by using a Bioanalyzer 2001 using an RNA 6000 Nano Kit (Agilent Technologies, Palo Alto, CA, USA) to achieve a $28 \mathrm{~S}: 18 \mathrm{~S}$ ratio of $1.2: 1$ to $2.2: 1$ and an RNA integrity number (RIN) above 7.

Partial HD-Zips III gene sequences were identified among EST (Expressed Sequenced Tags) obtained from the conifers P. taeda [49] and P. glauca [50] through BLAST analysis (Basic Local Alignment Search Tool). Completed coding sequences and untranslated 3' and 5' region (UTRs) were isolated by using PCR-cloning with degenerate primers or 3' RACE, 5' RACE (SMART RACE cDNA Amplification, BD Biosciences Clontech, Mountain View, CA, USA) or both cloning methods with mRNAs from needles or xylem. The cDNAs were cloned in pCR2.1 with the TA cloning kit (Invitrogen, Carlsbad, CA, USA), then sequenced with a 16-capillary genetic analyzer (ABI Prism 3130XL and an ABI Prism 3100XL, Applied Biosystems, Foster City, CA, USA). Public databases were searched by use of the BLAST algorithms to identify HD-Zip III sequences in the Poplar genome (JGI, P. trichocarpa v1.0, [26] and updated v2.0 http://www.phytozome.net/poplar), and the non-redundant NCBI database (nr; http://blast.ncbi.nlm. nih.gov/). Corresponding cDNAs were amplified from first-strand cDNA which had been derived from pooled total RNA extracted from developing secondary xylem, tips and young leaves $(P$. trichocarpa $\times P$. deltoides and $P$. tremula $\times P$. alba). The identity of each cDNA clone was confirmed by complete and partial sequence comparisons with the poplar genome and previously identified EST sequences [6].

The predicted HD-Zip III amino acid sequences from multiple species were aligned using ClustalW algorithm [51], which is included in the Bioedit software [52], and then refined manually. Phylogenetic analyses used the alignment of complete amino acid sequences via MEGA software [53]. A Neighbour-Joining phylogenetic tree [28] was constructed based on a Poisson correction model and pair-wise deletion algorithm (1,000 bootstrap replicates). The phylogenetic tree was rooted with three members of other HD-Zip class 1 and 4 sequences found in the Genbank database [54].

\section{RT-qPCR procedures}

Total RNA $(2 \mu \mathrm{g})$ was DNase-treated and reverse-transcribed using the SuperScriptII First-Strand Synthesis System for RT-PCR following manufacturer's recommendations (Invitrogen, Carlsbad, CA, USA). The resulting first-strand cDNA was diluted 1:10 in deionised water before quantitative PCR determinations. An aliquot of cDNA equivalent of $20 \mathrm{ng}$ of total RNA was used per $20 \mu \mathrm{L}$ of PCR reaction. Amplifications were performed in a QuantiTect ${ }^{\mathrm{Tm}} \mathrm{PCR} \mathrm{SYBR}^{\circ}$ Green Kit
(Qiagen, Mississauga, ON, Canada) with $0.3 \mu \mathrm{M}$ of $5^{\prime}$ and 3' primers, with a DNA Engine Opticon TM 2 System (MJ Research Inc., Ramsey, MN, USA) following the manufacturer's instructions. Primers were designed with Primer3 software [55], with a melting temperature (Tm) between $55^{\circ} \mathrm{C}$ and $62^{\circ} \mathrm{C}$, and produced amplicons between 100 and 250 bp (Additional file 1). The poplar RT-qPCR primers were tested on the clones H11-11 and INRA-717. Amplification reaction efficiencies were between 90 and 105\% for each primer pair. The thermal-cycling parameters were as follows: $95^{\circ} \mathrm{C}$ for 15 min; 45 cycles of $94^{\circ} \mathrm{C}$ for $15 \mathrm{sec}, 55^{\circ} \mathrm{C}$ for $1 \mathrm{~min}$ and $72^{\circ} \mathrm{C}$ for $40 \mathrm{sec}$; followed by a melting curve analysis from $54^{\circ} \mathrm{C}$ to $95^{\circ} \mathrm{C}$ with increments of $0.1^{\circ} \mathrm{C}$ per step to verify the specificity of the amplification and presence of primer dimers. The number of target copies in each sample was determined from Ct values (cycle threshold values) using a linearised plasmid or purified PCR product to produce a standard curve, which was obtained by averaging values from several runs. Two to six independent sample assays were performed and each sample was loaded in duplicate. Results were normalised relative to the absolute RNA used in a single reaction and with the transcript level of a reference gene. The spruce data were normalised to the reference of transcript level gene encoding Elongation factor 1 alpha, EF1a, (AJ132534) [56]. The EF1a gene were strongly expressed and showed low variation between spruce tissues [57]. In poplar, the reference was a cell division cycle 2 gene homologue, CDC2 (AF194820) [58]. CDC2 cycle threshold values $(\mathrm{Ct})$ averaged $18.5+0.5$ (Standard Deviation), which is the experimental error range of the RT-qPCR cycler device. For statistical analyses of the RT-qPCR data from auxin-related treatments and transgenic experiments (Tables $1,2,3$ ) a $\log _{2}$ transformation was applied to the number of target copies.

\section{Agrobacterium transformation and growth of transgenic poplars}

Over-expression constructs were obtained by inserting the complete coding sequences of PtaHB1 and PtaHB7 from $P$. tremula $\times P$. alba clone INRA-717 between the maize ubiquitin promoter [59] and a $35 \mathrm{~S}$ terminator into the pCambia1305.2 vector http://www.cambia.org. The resulting plasmids were then transferred into $A$. tumefaciens strain C58 pGV2260 [60].

For transformation, in vitro plantlets of the hybrid poplar clone INRA-717 were micropropagated on hormone-free 1/2 MS medium [61] supplemented with vitamin D3. Internodes from in vitro plantlets were cocultivated with the engineered A. tumefaciens according to the method of Leple et al. (1992) [62], with the following modifications. After co-cultivation, the explants were decontaminated of $A$. tumefaciens with cefotaxim 
and transferred onto M2 medium containing cefotaxim alone [62]. Transgenic calluses were selected on M3 medium supplemented with hygromycin $\left(10 \mathrm{mg} \mathrm{L}^{-1}\right)$ and emerging shoots were transferred for rooting to $1 / 2$ MS medium without hygromycin, screened for positive $\mathrm{X}$-glucuronidase activity [62], and assayed for poplar PtaHB1 and PtaHB7 mRNA accumulation by RT-qPCR. Shoots with elevated PtaHB1 and PtaHB7 mRNA levels relative to WT control were transferred to the greenhouse where they were grown under a photoperiod of $16 \mathrm{~h}$ of light, at $24 / 20^{\circ} \mathrm{C}$ (day/night). For each construct, four to six trees for each of six lines (independent transformation events), and 10 to 15 WT INRA-717 trees were propagated and carried forward together for phenotypic determinations.

\section{Phenotype characterisations of transgenic poplars}

Morphometric measurements were performed on two trees from three independent transformation events per line and six control trees (WT). Internode length, petiole length, and petiole angle were measured from LPI 1 to the bottom of each tree. Vascular tissue organisation was visualised on samples taken from fresh stem and petiole sections from LPI 20. Samples were fixed in $4 \%$ paraformaldehyde - $2 \%$ glutaraldehyde cacodylate buffer ( $\mathrm{pH}$ 7.2) for 12 hours under vacuum, and stored at $4^{\circ} \mathrm{C}$ before embedding in paraffin blocks. Microtome cross-sections $(10 \mu \mathrm{m}$ thick) were mounted on glass slides; after the paraffin was removed, the sections were rehydrated for observation under UV light on an Olympus BX51 microscope (Olympus, Montreal, QC, Canada). Fibre and vessel distributions in petioles were visualised by lignin autofluorescence and counted (75 to 150 cells per field imaged). The average fibre to vessel ratio was computed from four transgenic plants and four WT plants. Fibre Quality Analysis (FQA; OpTest Equipment, Hawkesbury, ON, Canada) used freshly debarked internodes, and intact petiole sections from LPI 6, LPI 21 and LPI 43. The samples were macerated in Franklin's solution until they were completely bleached and the fibres could be separated [63]. The FQA weighted, fibre length (lw) data (8000 individual fibres per petiole or internode) were obtained from three trees per transgenic line on six lines and on six control trees. For analyses, the data were placed into bins of 0.10 to $0.25 \mathrm{~mm}$.

\section{Statistical analyses of phenotypic and RT-qPCR data}

Statistical treatment of phenotypic data compared transgenic poplars transformed empty vector (control lines) and those transformed with the PtaHB1 gene construct. Except where otherwise noted, the data were not transformed and each tree was considered an individual experiment unit. Normality was confirmed and Student's $t$ tests were used for petiole length and angle data (Figure 3), with each internode tested separately in SYSTAT 13 (Cranes Software International Ltd., Chicago, IL). Student's $t$ tests were also used to compare the proportion fibres to vessels within the petioles, determined by using UV microscopy (Figure 4A and 4B). The fibre length data (FQA) were analysed by comparing the proportion (\%) of fibres in each length class (bin) (Figure 4C) with a one-way ANOVA, on each bin independently, and also by comparing the overall fibre length data (Figure 4D). Before analysis the normality of the data was confirmed, and ANOVAs were carried out using procGLM in SAS (Version 9.01, SAS Institute Inc., Cary, NC).

For RT-qPCR data comparing control and PtaHB1 transgenic lines (Table 1,2) and comparing the impact auxin-related treatments (Table 3) normality and Student's $t$ tests were applied to $\log _{2}$ transformed numbers of transcript targets in SYSTAT 13 (Cranes Software International Ltd., Chicago, IL).

\section{Microarray RNA profiling}

A poplar $3.4 \mathrm{~K}$ cDNA microarray was prepared by the ARBOREA project and is described in Pitre et al. (2010) [64]. RNA transcript profiling was carried for each of the transgenic constructs, with four samples from two independent transformation events compared with four control samples from two independent transformation events (empty vector controls). Primary stem tissues (LPI 0 to 5) were used for profiling PtaHB1 over-expressing lines and secondary stem xylem tissues (LPI 21 to 43) were used for the PtaHB7. The microarray hybridization methods were as described in Pitre et al. (2010) [64]. Each microarray hybridisation used $1 \mu \mathrm{g}$ of total RNA that was amplified using the SuperScript ${ }^{\text {mix }}$ Indirect RNA Amplification System (Invitrogen, Carlsbad, CA, USA) and $5 \mu \mathrm{g}$ of the aRNA were labelled with Alexa Fluor ${ }^{\circ} 555$ and 647 dyes (Invitrogen Carlsbad, CA, USA), for use in dye-swap experiments. The poplar $3.4 \mathrm{~K}$ microarray slides were pre-hybridised for 2 hours at $42^{\circ}$ $\mathrm{C}$ in a solution containing $5 \times$ SSC, $0.1 \%$ SDS, $0.02 \%$ BSA (w/v), 0.01\% herring sperm DNA (w/v), and $50 \%$ formamide. The slides were then washed twice in $0.1 \times$ SSC, once in water, rinsed in 2-propanol, and finally dried by centrifugation. The labelled targets $(3.5 \mu \mathrm{L})$ were mixed with $52.5 \mu \mathrm{L}$ of hybridisation solution containing $5 \times$ SSC, $0.1 \%$ SDS, $0.01 \%$ herring sperm DNA $(\mathrm{w} / \mathrm{v})$, and $50 \%$ deionised formamide. The mixture was heat-denatured for $4 \mathrm{~min}$ at $95^{\circ} \mathrm{C}$ and cooled for $5 \mathrm{~min}$ on ice prior to hybridisation to the microarray. The microarray was then covered with a LifterSlip (Erie Scientific Company, Portsmouth, NH, USA) and placed in a hybridisation chamber II with increased depth 
(Corning, Lowell, MA, USA) and incubated for $12 \mathrm{~h}$ at $45^{\circ} \mathrm{C}$ in a model 1012 hybridisation oven (Shel Lab, Cornelius, OR, USA). After hybridisation, the slides were iteratively washed for $15 \mathrm{~min}$ in $2 \times$ SSC $+0.5 \%$ SDS, $0.5 \times \mathrm{SSC}+0.1 \%$ SDS and $0.1 \times \mathrm{SSC}$ solutions at $45^{\circ} \mathrm{C}$.

The slides were scanned using a ScanArray ${ }^{\mathrm{m}}$ Express scanner (Packard BioScience, Meriden, CT, USA) and the image files were analysed using QuantArray ${ }^{\circ}$ software (Packard BioScience, Meriden, CT, USA). Scan intensities were comparable between sets of slides for a given hybridisation. Data analysis was carried out using Bioconductor packages http://www.bioconductor.org distributed in R ( $R$ Development Core Team 2008). Median foreground intensity minus median background intensity was the response variable used for the statistical analysis. Data quality was assessed using graphical analysis tools in the marray and olin packages available in Bioconductor, and by assessment of within- and between-slide Pearson product-moment correlation coefficients $(r)$, which were calculated both from the raw intensities and after normalisation. The composite normalisation method [65] was applied by using the two functions maNorm $2 D$ and maNormLoess in the marray package [66]. We identified differentially expressed sequences with the SAM package release 3.0 [31] using a false discovery rate (FDR) [30] threshold set to $5 \%$ ( $q$ $<5.00$ ). Data reported in the article are $\log _{2}$-ratios of Alexa Fluor ${ }^{\circ} 555 / 647$, which are denoted M. Raw data are available in the Gene Expression Omnibus (GEO) database (accession number: GSE24703, http://www. ncbi.nlm.nih.gov/geo/).

\section{Additional material}

Additional file 1: Gene predicted names, accession numbers and primers for RT-qPCR used in poplar and white spruce. First column contains GenBank accession number, second column contains name of the genes cloned in poplar (POPTR_ID V2 of Populus trichocarpa) and white spruce, third column contains RT-qPCR primers forward and reverse.

\section{List of abbreviations}

EST: expressed sequence tag; HD-ZIP: homeodomain leucine-zipper; RTQPCR: reverse-transcription quantitative PCR;

\section{Acknowledgements}

The authors thank Mr. Jeffrey Stott, Dr. Rob Holt and Dr. Marco Marra (Genome Sciences Centre, Vancouver, BC) for EST sequencing. Dr. Charles Paule (Univ. of Minnesota), Dr. Nathalie Pavy, Mr Hugo Bérubé, Dr. Brian Boyle and Mr Sébastien Caron (Univ. Laval) for bioinformatics and assistance with microarray development and analysis methods, Dr. Daniel Tessier and Ms. Tracy Rigby (Biotechnology Research Institute, NRC, Montreal, QC) for production of the microarray, and Dr. Bill Parsons (Univ. of Sherbrooke) and Dr. Louis Bernatchez (Univ. Laval) for manuscript reviewing. Financial support was received from Genome Canada and Genome Québec for the Arborea project (AS, JM), from the Fonds Québécois de Recherche sur la Nature et les Technologies (FQRNT, JM).

\section{Author details}

'Département des Sciences du Bois et de la Forêt, Université Laval, 2405 rue de la Terrasse, Québec, QC, G1V 0A6, Canada. ²aurentian Forestry Centre, 1055 rue du P.E.P.S., Québec, QC, G1V 4C7, Canada. ${ }^{3}$ BEI, Joint BioEnergy Institute, 5885 Hollis St, 4th floor, Emeryville, CA 94608, USA. ${ }^{4}$ Department of Biological Sciences, University of Alberta, Edmonton, AB, T6G 2E9, USA.

\section{Authors' contributions}

CLC drafted the manuscript, carried out the poplar sequence analyses, the phylogenetic tree construction, RT-qPCR analyses in poplar, transgenic poplar characterisations and microarrays results interpretations. FM carried out the spruce sequence analyses and RT-qPCR analyses in spruce. VR conducted the microarray RNA-profiling experiment. MO isolated PtrHB1 and PtrHB5 CDNA sequences. $\mathrm{CL}$ carried out plant tissue transformations and produced the transgenic trees. M-JM prepared vector plant tissue transformation. JEKC oversaw microarray development, participated in the manuscript revision. AS oversaw plant tissue transformations and production of transgenic trees, participated in the manuscript revision. JJM oversaw overall project and manuscript preparation. All authors read and approved the final manuscript.

Received: 4 June 2010 Accepted: 11 December 2010

Published: 11 December 2010

\section{References}

1. Fukuda H: Xylogenesis: Initiation, progression, and cell death. Annual Review of Plant Physiology and Plant Molecular Biology 1996, 47:299-325.

2. Telewski FW, Aloni R, Sauter JJ: Physiology of secondary tissues of Populus. In Biology of Populus and its implications for management and conservation. Edited by: Stettler RF, Bradshaw HD, Heilan PE, Hinckley TM. Ottawa, ON, Canada: NRC Research Press; 1996:301-329.

3. Zhang SY: Effect of growth-rate on wood specific-gravity and selected mechanical-properties in individual-species from distinct wood categories. Wood Science and Technology 1995, 29:451-465.

4. Izawa T, Takahashi Y, Yano M: Comparative biology comes into bloom: genomic and genetic comparison of flowering pathways in rice and Arabidopsis. Current Opinion in Plant Biology 2003, 6:113-120.

5. Nieminen KM, Kauppinen L, Helariutta Y: A weed for wood? Arabidopsis as a genetic model for xylem development. Plant Physiology 2004, 135:653-659

6. Ko JH, Prassinos C, Han KH: Developmental and seasonal expression of PtaHB1, a Populus gene encoding a class III HD-Zip protein, is closely associated with secondary growth and inversely correlated with the level of microRNA (miR166). New Phytologist 2006, 169:469-478.

7. Aso K, Kato M, Banks JA, Hasebe M: Characterization of homeodomainleucine zipper genes in the fern Ceratopteris richardii and the evolution of the homeodomain-leucine zipper gene family in vascular plants. Molecular Biology and Evolution 1999, 16:544-552.

8. Johannesson H, Wang Y, Engstrom P: DNA-binding and dimerization preferences of Arabidopsis homeodomain-leucine zipper transcription factors in vitro. Plant Molecular Biology 2001, 45:63-73.

9. Ohashi-lto K, Kubo M, Demura T, Fukuda H: Class III homeodomain leucine-zipper proteins regulate xylem cell differentiation. Plant and Cell Physiology 2005, 46:1646-1656.

10. Mukherjee $K$ and TR Burglin: MEKHLA, a novel domain with similarity to PAS domains, is fused to plant homeodomain-leucine zipper III proteins. Plant Physiology 2006, 140:1142-1150.

11. Chandler JW, Cole M, Flier A, Grewe B, Werr W: The AP2 transcription factors DORNROSCHEN and DORNROSCHEN-LIKE redundantly control Arabidopsis embryo patterning via interaction with PHAVOLUTA. Development 2007, 134:1653-1662.

12. Zhong $\mathrm{RQ}$, Ye ZH: Alteration of auxin polar transport in the Arabidopsis ifl1 mutants. Plant Physiology 2001, 126:549-563.

13. Zhong $R Q$, Ye $Z H$ : Amphivasal vascular bundle 1, a gain-of-function mutation of the IFL1/REV gene, is associated with alterations in the polarity of leaves, stems and carpels. Plant and Cell Physiology 2004, 45:369-385.

14. Ohashi-Ito $K$, Demura T, Fukuda H: Promotion of transcript accumulation of novel Zinnia immature xylem-specific HD-Zip III homeobox genes by brassinosteroids. Plant and Cell Physiology 2002, 43:1146-1153. 
15. McConnell JR, Emery J, Eshed Y, Bao N, Bowman J, Barton MK: Role of PHABULOSA and PHAVOLUTA in determining radial patterning in shoots. Nature 2001, 411:709-713.

16. Baima S, Nobili F, Sessa G, Lucchetti S, Ruberti I, Morelli G: The expression of the Athb-8 homeobox gene is restricted to provascular cells in Arabidopsis thaliana. Development 1995, 121:4171-4182.

17. Baima S, Possenti M, Matteucci A, Wisman E, Altamura MM, Ruberti I, Morelli G: The Arabidopsis ATHB-8 HD-Zip protein acts as a differentiation-promoting transcription factor of the vascular meristems. Plant Physiology 2001, 126:643-655.

18. Prigge MJ, Otsuga D, Alonso JM, Ecker JR, Drews GN, Clark SE: Class III homeodomain-leucine zipper gene family members have overlapping, antagonistic, and distinct roles in Arabidopsis development. Plant Cell 2005, 17:61-76.

19. Emery JF, Floyd SK, Alvarez J, Eshed Y, Hawker NP, Izhaki A, Baum SF, Bowman JL: Radial patterning of Arabidopsis shoots by class III HD-ZIP and KANADI genes. Current Biology 2003, 13:1768-1774.

20. Cronk QCB: Plant eco-devo: the potential of poplar as a model organism. New Phytologist 2005, 166:39-48.

21. Floyd SK, Zalewski CS, Bowman JL: Evolution of class III homeodomainleucine zipper genes in streptophytes. Genetics 2006, 173:373-388.

22. Prigge MJ, Clark SE: Evolution of the class III HD-Zip gene family in land plants. Evolution \& Development 2006, 8:350-361.

23. Degroote DK, Larson PR: Correlations between net auxin and secondary xylem development in young Populus-deltoides. Physiologia Plantarum 1984, 60:459-466.

24. Ingouff $M$, Farbos I, Lagercrantz $U$, von Arnold S: PaHB1 is an evolutionary conserved HD-GL2 homeobox gene expressed in the protoderm during Norway spruce embryo development. Genesis 2001, 30:220-230.

25. Namroud M-C, Guillet-Claude C, MacKay J, Isabel N, Bousquet J: Molecular evolution of five regulatory genes in the conifer Picea: evidence for selection and large-scale demographic changes. Molecular Evolution 2010.

26. Tuskan GA, DiFazio S, Jansson S, Bohlmann J, Grigoriev I, Hellsten U, Putnam N, Ralph S, Rombauts S, Salamov A, Schein J, Sterck L, Aerts A, Bhalerao RR, Bhalerao RP, Blaudez D, Boerjan W, Brun A, Brunner A, Busov V, Campbell M, Carlson J, Chalot M, Chapman J, Chen GL, Cooper D, Coutinho PM, Couturier J, Covert S, Cronk Q, Cunningham R, Davis J, Degroeve S, Dejardin A, Depamphilis C, Detter J, Dirks B, Dubchak I, Duplessis S, Ehlting J, Ellis B, Gendler K, Goodstein D, Gribskov M, Grimwood J, Groover A, Gunter L, Hamberger B, Heinze B, Helariutta Y, Henrissat B, Holligan D, Holt R, Huang W, Islam-Faridi N, Jones S, JonesRhoades $M$, Jorgensen $R$, Joshi $C$, Kangasjarvi J, Karlsson J, Kelleher C, Kirkpatrick R, Kirst M, Kohler A, Kalluri U, Larimer F, Leebens-Mack J, Leple JC, Locascio P, Lou Y, Lucas S, Martin F, Montanini B, Napoli C, Nelson DR, Nelson C, Nieminen K, Nilsson O, Pereda V, Peter G, Philippe R, Pilate G, Poliakov A, Razumovskaya J, Richardson P, Rinaldi C, Ritland K, Rouze P, Ryaboy D, Schmutz J, Schrader J, Segerman B, Shin H, Siddiqui A Sterky F, Terry A, Tsai CJ, Uberbacher E, Unneberg P, Vahala J, Wall K, Wessler S, Yang G, Yin T, Douglas C, Marra M, Sandberg G, Van de Peer Y, Rokhsar D: The genome of black cottonwood, Populus trichocarpa (Torr. \& Gray). Science 2006, 313:1596-1604

27. Chan RL, Gago GM, Palena CM, Gonzalez DH: Homeoboxes in plant development. Biochimica \& Biophysica Acta-Gene Structure and Expression 1998, 1442:1-19.

28. Saitou N, Nei M: The neighbor-joining method: A new method for reconstructing phylogenetic trees. Molecular Biology \& Evolution 1987, 4:406-425

29. Jansson J, and Douglas CJ: Populus: A model system for plant biology. Annual Review of Plant Biology 2007, 58:435-458.

30. Benjamini $Y$, Hochberg $Y$ : Controlling the false discovery rate - a practical and powerful approach to multiple testing. Journal of the Royal Statistical Society Series B-Methodological 1995, 57:289-300.

31. Efron B, Tibshirani R, Storey JD, Tusher V: Empirical Bayes analysis of a microarray experiment. Journal of the American Statistical Association 2001, 96:1151-1160.

32. Rise ML, Jones SRM, Brown GD, von Schalburg KR, Davidson WS, Koop BF: Microarray analyses identify molecular biomarkers of Atlantic salmon macrophage and hematopoietic kidney response to Piscirickettsia salmonis infection. Physiological Genomics 2004, 20:21-35.
33. Morey JS, Ryan JC, Van Dolah FM: Microarray validation: Factors influencing correlation between oligonucleotide microarrays and realtime PCR. Biological Procedures Online 2006, 8:175-193.

34. Whippo CW, Hangarter RP: A brassinosteroid-hypersensitive mutant of BAK1 indicates that a convergence of photomorphogenic and hormonal signaling modulates phototropism. Plant Physiology 2005, 139:448-457.

35. Lafarguette F, Leple JC, Dejardin A, Laurans F, Costa G, LesageDescauses MC, Pilate G: Poplar genes encoding fasciclin-like arabinogalactan proteins are highly expressed in tension wood. New Phytologist 2004, 164:107-121.

36. Espartero J, Sanchez Aguayo I, Pardo JM: Molecular characterization of glyoxalase-I from a higher plant: Upregulation by stress. Plant Molecular Biology 1995, 29:1223-1233.

37. Galichet A, Gruissem W: Developmentally controlled farnesylation modulates AtNAP1; 1 functions in cell proliferation and cell expansion during Arabidopsis leaf development. Plant Physiology 2006, 142:1412-1426

38. Anterola AM, Lewis NG: Trends in lignin modification: a comprehensive analysis of the effects of genetic manipulations/mutations on lignification and vascular integrity. Phytochemistry 2002, 61:221-294.

39. Xiang $S$, Usunow $G$, Lange $G$, Bush $M$, Tong L: Crystal structure of 1deoxy-D-xylulose 5-phosphate synthase, a crucial enzyme for isoprenoids biosynthesis. Journal of Biological Chemistry 2007, 282:2676-2682.

40. Loivamaki M, Louis S, Cinege G, Zimmer I, Fischbach RJ, Schnitzler JP Circadian rhythms of isoprene biosynthesis in Grey poplar leaves. Plant Physiology 2007, 143:540-551.

41. Sakakibara K, Nishiyama T, Kato M, Hasebe M: Isolation of homeodomainleucine zipper genes from the moss Physcomitrella patens and the evolution of homeodomain-leucine zipper genes in land plants. Molecular Biology and Evolution 2001, 18:491-502.

42. Devos KM, Brown JKM, Bennetzen JL: Genome size reduction through illegitimate recombination counteracts genome expansion in Arabidopsis. Genome Research 2002, 12:1075-1079.

43. Zhong $\mathrm{RQ}$, Ye ZH: IFL1, a gene regulating interfascicular fibre differentiation in Arabidopsis, encodes a homeodomain-leucine zipper protein. Plant Cell 1999, 11:2139-2152.

44. Otsuga D, DeGuzman B, Prigge MJ, Drews GN, Clark SE: REVOLUTA regulates meristem initiation at lateral positions. Plant Journal 2001 25:223-236.

45. Kim J, Jung JH, Reyes JL, Kim YS, Kim SY, Chung KS, Kim JA, Lee M, Lee Y, Kim VN, Chua NH, Park CM: microRNA-directed cleavage of ATHB15 mRNA regulates vascular development in Arabidopsis inflorescence stems. Plant Journal 2005, 42:84-94.

46. Zhong RQ, Taylor JJ, Ye ZH: Transformation of the collateral vascular bundles into amphivasal vascular bundles in an Arabidopsis mutant. Plant Physiology 1999, 120:53-64.

47. Larson PR, Isebrands JG: The plastochron index as applied to developmental studies of cottonwood. Canadian Journal of Forest Research 1971, 1:1-11.

48. Chang S, Puryear J, Cairney J: A simple and effcient method for isolating RNA from pine trees. Plant Molecular Biology Reporter 1993, 11:113-116.

49. Kirst M, Myburg AA, De Leon JPG, Kirst ME, Scott J, Sederoff R: Coordinated genetic regulation of growth and lignin revealed by quantitative trait locus analysis of cDNA microarray data in an interspecific backcross of Eucalyptus. Plant Physiology 2004, 135:2368-2378.

50. Pavy N, Paule C, Parsons L, Crow JA, Morency MJ, Cooke J, Johnson JE, Noumen E, Guillet-Claude C, Butterfield Y, Barber S, Yang G, Liu J, Stott J, Kirkpatrick R, Siddiqui A, Holt R, Marra M, Seguin A, Retzel E, Bousquet J, MacKay J: Generation, annotation, analysis and database integration of 16,500 white spruce EST clusters. BMC Genomics 2005, 6:144.

51. Chenna R, H Sugawara, T Koike R, Lopez T, Gibson TJ, Higgins DG, Thompson JD: Multiple sequence alignment with the Clustal series of programs. Nucleic Acids Research 2003, 31:3497-3500.

52. Hall TA, Hall N: BioEdit: a user-friendly biological sequence alignment editor and analysis program for Windows 95/98/NT. Nucleic Acids Symposium Series 1999, 41:95-99.

53. Kumar S, Nei M, Dudley J, Tamura K: MEGA: A biologist-centric software for evolutionary analysis of DNA and protein sequences. Briefings in Bioinformatics 2008, 9:299-306. 
54. Benson D A, Karsch-Mizrachi I, Lipman DJ, Ostell J, Wheeler DL: GenBank. Nucleic Acids Research 2005, 33:D34-D38.

55. Rozen S, Skaletsky H: Primer3 on the WWW for general users and for biologist programmers. Methods in Molecular Biology 2003, 132:365-386.

56. Pulikowska J, Twardowski T: The elongation factor 1 from wheat germ: structural and functional properties. Acta Biochimica Polonica 1982, 29:245-258.

57. Bedon F, Grima-Pettenati J, Mackay J: Conifer R2R3-MYB transcription factors: sequence analyses and gene expression in wood-forming tissues of white spruce (Picea glauca). BMC Plant Biology 2007, 7:7-17.

58. Le Bail A, Dittami SM, de Franco PO, Rousvoal S, Cock MJ, Tonon T, Charrier B: Normalisation genes for expression analyses in the brown alga model Ectocarpus siliculosus. BMC Molecular Biology 2008, 9:75

59. Christensen AH, Sharrock RA, Quail PH: Maize polyubiquitin genes Structure, thermal perturbation of expression and transcript splicing, and promoter activity following transfer to protoplasts by electroporation. Plant Molecular Biology 1992, 18:675-689.

60. Hellens RP, Edwards EA, Leyland NR, Bean S, Mullineaux PM: pGreen: a versatile and flexible binary Ti vector for Agrobacterium-mediated plant transformation. Plant Molecular Biology 2000, 42:819-832.

61. Mukherjee K, Burglin TR: MEKHLA, a novel domain with similarity to PAS domains, is fused to plant homeodomain-leucine zipper III proteins. Plant Physiology 2006, 140:1142-1150.

62. Leple JC, Brasileiro ACM, Michel MF, Delmotte F, Jouanin L: Transgenic poplars - Expression of chimeric genes using 4 different constructs. Plant Cell Reports 1992, 11:137-141.

63. Franklin GL: Preparation of thin sections of synthetic resins and woodresin composites and a new macerating method for wood. Nature 1945, 155:51.

64. Pitre FE, Lafarguette F, Boyle B, Pavy N, Caron S, Dallaire N, Poulin PL, Ouellet M, Morency MJ, Wiebe N, Lim EL, Urbain A, Mouille G, Cooke JEK, Mackay JJ: High nitrogen fertilization and stem leaning have overlapping effects on wood formation in poplar but invoke largely distinct molecular pathways. Tree Physiology 2010.

65. Yang YH, Dudoit S, Luu P, Lin DM, Peng V, Ngai J, Speed TP: Normalization for CDNA microarray data: a robust composite method addressing single and multiple slide systematic variation. Nucleic Acids Research 2002, 30:4.

66. Dudoit S, Yang YH, Callow MJ, Speed TP: Statistical methods for identifying differentially expressed genes in replicated CDNA microarray experiments. Statistica Sinica 2002, 12:111-139.

doi:10.1186/1471-2229-10-273

Cite this article as: Côté et al:: Gene family structure, expression and functional analysis of HD-Zip III genes in angiosperm and gymnosperm forest trees. BMC Plant Biology 2010 10:273.

\section{Submit your next manuscript to BioMed Central and take full advantage of:}

- Convenient online submission

- Thorough peer review

- No space constraints or color figure charges

- Immediate publication on acceptance

- Inclusion in PubMed, CAS, Scopus and Google Scholar

- Research which is freely available for redistribution

Submit your manuscript at www.biomedcentral.com/submit
Biomed Central 